\title{
Signs of ultra-high-energy electrons emission with energy up to 105 times of beam energy are detected at downstream of the RF cavity of storage ring
}

Dapeng Qian ( $\sim$ ykqdpnew@163.com )

Retired

\section{Article}

Keywords: the extended Einstein-Lorentz mass formula, ultra-high energy electron, electron storage ring, electromagnetic calorimeter

Posted Date: February 17th, 2021

DOl: https://doi.org/10.21203/rs.3.rs-214987/v1

License: (c) (1) This work is licensed under a Creative Commons Attribution 4.0 International License.

Read Full License 


\title{
Signs of abnormal ultra-high-energy electrons emission with energy up to $10^{5}$ times of the beam energy are detected at downstream of the RF cavity of the electron storage ring
}

\author{
Dapeng Qian
}

\begin{abstract}
In a new version of special relativity that absorbed the uncertainty principle, the Einstein-Lorentz mass formula proved to be a special case of a more universal equation. The new equation indicates that there is a "high speed but low mass" weak effect in particle motion, which will cause the generation of abnormal ultra-high energy electrons with a small probability when an electron beam passes through an accelerating electric field. The author used the method of long-times accumulation detection to test it on the BEPCII, which results show that there is indeed emission of abnormal electrons with energy up to $10^{5}$ times of the beam energy at the downstream of the RF cavity of the electron storage ring. Therefor, it is suggested to use the detector with an online real-time display function, such as the "Shashlyk calorimeter", to detect the single event of ultra-high energy electron, so to fully verify this previously unknown phenomenon and further discover new physics.
\end{abstract}

Keywords: the extended Einstein-Lorentz mass formula, ultra-high energy electron, electron storage ring, electromagnetic calorimeter.

\section{Introduction}

How to unify the relativity and quantum mechanics? This is a big problem of physics, it has always attracted much attention. The popular view is that with the establishment of Dirac equation and quantum electrodynamics, the task of unifying special relativity and quantum mechanics has completed, and the remaining work is only to solve the unified problem of the general relativity and quantum mechanics.

However, if we take a closer look, we will find that the above view point is flawed that in quantum electrodynamics the so-called unification of special relativity and quantum mechanics is actually the former's unilateral "invasion" to the latter, the two have not achieved mutual integration in basic concepts.

Also, for many years, there has been extensive discussion on the issue of "Lorentz invariance violation" from the field of ultra-high-energy cosmic rays to the field of condensed matter physics, but theoretical physicists never touched on the root cause. Such a situation also remind people that the most important work at the moment is not to patch up some individual results of quantum field theory, but to consider how to make the special relativity to obey the general requirements of quantum mechanics in order to find what was left out at the source.

As we all know, in a fundamental sense, the special relativity is firstly a measurement theory on physical quantities such as time, space, energy, momentum, that is, a physical framework theory that makes principled norms for all measurement behaviors. On the other hand, we also know that the measurements to these physical quantities must also be limited by an insurmountable natural law, that is the Heisenberg uncertainty principle that reflects the essential characteristics of quantum mechanics. It is judged in this sense that the special relativity as a "clean" space-time measurement theory totally disregarding the uncertainty relationship cannot be a ultimate complete theory. Therefore, if the universal constraint of the uncertainty principle is taken into account, the special relativity must be further improved, and only after this work is completed can we truly move towards the full the unification of quantum mechanics and all theories of relativity. 
In fact, people have long realized that any complete physical theory about time and space must include not only the overall content of relativity but also the uncertainty principle, and the uncertainty principle must be regarded as one of the foundations of this theory. However, what method can be used to insert naturally the uncertainty relationship into the conceptual system of special relativity? To this problem, physics must give a clear answer.

The author provided a solution to this problem ${ }^{[1]}$. First, based on the unity nature of time and space established by relativity, as well as the spherical symmetry of three-dimensional space, the translation symmetry of time, and the uncertainty principle, a four-dimensional space-time cylinder model is established for quark and lepton. Then use this model to derive a series of new equations that can cover the old equations of special relativity and meet the requirements of the uncertainty principle, thereby to establish a new version of special relativity including the uncertainty principle, which achieves the unification of the special relativity and quantum mechanics in deeper level within a different way from quantum electrodynamics.

The most important one in new equations is the improved mass-speed relationship, also as "Extended Einstein-Lorentz mass formula". It reveals that concepts of the mass and energy have richer connotations, which not only be used to resolve some outstanding problems but also predicts new effects that can be tested experimentally. This article reports an experimental verification to one of predictions of the extended Einstein-Lorentz mass formula.

\section{Theory brief description: the extended Einstein-Lorentz mass formula and its prediction}

In a new version of the special relativity that absorbed the uncertainty principle, the EinsteinLorentz mass formula proved to be a special case of a more universal equation ${ }^{[1]}$. When the speed $0 \leq u \leq u_{d}=\left(1-4.64 \times 10^{-39}\right) c$, the extended Einstein-Lorentz mass formula is

$$
m=\frac{m_{0}\left(3 \zeta^{2}-2 \zeta^{3}\right)}{\sqrt{1-\frac{u^{2}}{c^{2}}}}
$$

Where $\zeta$ is a dimensionless random variable, $\quad 0 \leq \zeta \leq 1$

When $\zeta=1$ Eq. (1) has the maximum form, that is the well-known Einstein-Lorentz mass formula:

$$
m_{R}=\frac{m_{0}}{\sqrt{1-\frac{u^{2}}{c^{2}}}}
$$

When $\zeta<1, m=m_{R}\left(3 \zeta^{2}-2 \zeta^{3}\right)<m_{R}$, so the particles will be in an abnormal high speed but low mass (HSLM) state. The probability $P_{S}$ in this state depends on speed, that is, depends on the "Einstein-Lorentz energy $E_{R}\left(=m_{R} c^{2}\right)$ " that corresponds alone to the speed:

$$
P s=\frac{2 \sqrt{E_{R}^{2}-E_{0}^{2}}}{\Phi E_{0}+\sqrt{E_{R}^{2}-E_{0}^{2}}}
$$

Where $E_{0}$ is the rest energy of particle, $\Phi$ is a dimensionless constant,

$$
\Phi=1.037 \times 10^{19}
$$

Because $\Phi E_{0}$ is a huge value, in accelerator experiments of $E_{R}<<\Phi E_{0}$, the probability that particles appear in HSLM state is very small. Such as the electron's $\Phi E_{0}=5.3 \times 10^{15} \mathrm{GeV}$, but hitherto there are only $E_{R} \leq 10^{2} \mathrm{GeV}$ on all electron accelerators, so $P_{S}<4 \times 10^{-14}$ is calculated by Eq.(4). However, the probability of particles appear at the normal state of $\zeta=1$ (i.e. $m=m_{R}$ and $E=E_{R}$ ) is

$$
P_{R}=1-P_{S}
$$


Obviously, in the current experimental energy region, the $E=E_{R}$ state is the most probability state, and the classical Einstein-Lorentz formula (3) describes only the mass (energy)-speed relationship under this state.

In short, when the speed is constant, according to $\zeta=1,1>\zeta>0, \zeta=0$ the particles can appear in various states of $m=m_{R}, m_{R}>m>0, m=0$. In the most probable state of $m=m_{R}$, the Lorentz invariance exists; in the abnormal $m_{R}>m \geq 0$ state, the Lorentz invariance violation. The so-called "quasi-particles" in the study of condensed matter physics, that is, the portrayal of a large number of electronic collective behaviors that violates Lorentz invariance in the interaction system, reflect in fact the different $\zeta$ states of real electrons, and especially the fermion of zero mass reflects the behavior of electrons in the state of $\zeta=0$.

The experiments reported in this paper are designed for the electron beam of the accelerator, which purpose is to find the abnormal phenomenon caused by the HSLM effect in $\zeta<1$ state, so as to test the extended Einstein-Lorentz mass formula.

\section{Experimental detection to a previously unknown phenomena predicted by the theory}

\subsection{Experimental principle}

According to Eq.(1), among a large number of electrons moving at a same speed, the mass $m$ of electron at the state of $\zeta<1$ is smaller than the mass $m_{R}$ of electron at the normal state of $\zeta=1$, that is, $m=m_{R}\left(3 \zeta^{2}-2 \zeta^{3}\right)<m_{R}$. Therefore, in the electron storage ring, when the electron beam passes the accelerating electric field, the electrons at the state of $\zeta<1$ will be faster accelerated than the normal electrons of the same speed. After leaving the accelerating electric field, these abnormal electrons will return to the most probable $\zeta=1$ state. At this time, their energy $E_{R}{ }^{*}$ will be much higher than the energy of normal electrons, and some abnormal electrons with particularly high $E_{R}{ }^{*}$ will inject into the copper blind end and cause abnormal electromagnetic showers there.

Therefore, a detector such as an electromagnetic calorimeter is placed outside the copper blind end of downstream of RF cavity to collect the electromagnetic shower. If electromagnetic shower with theoretically expected features caused by the ultra-high energy electrons are found in various background radiations, the predicted HSLM effect will obtain a verification (See Fig.1).

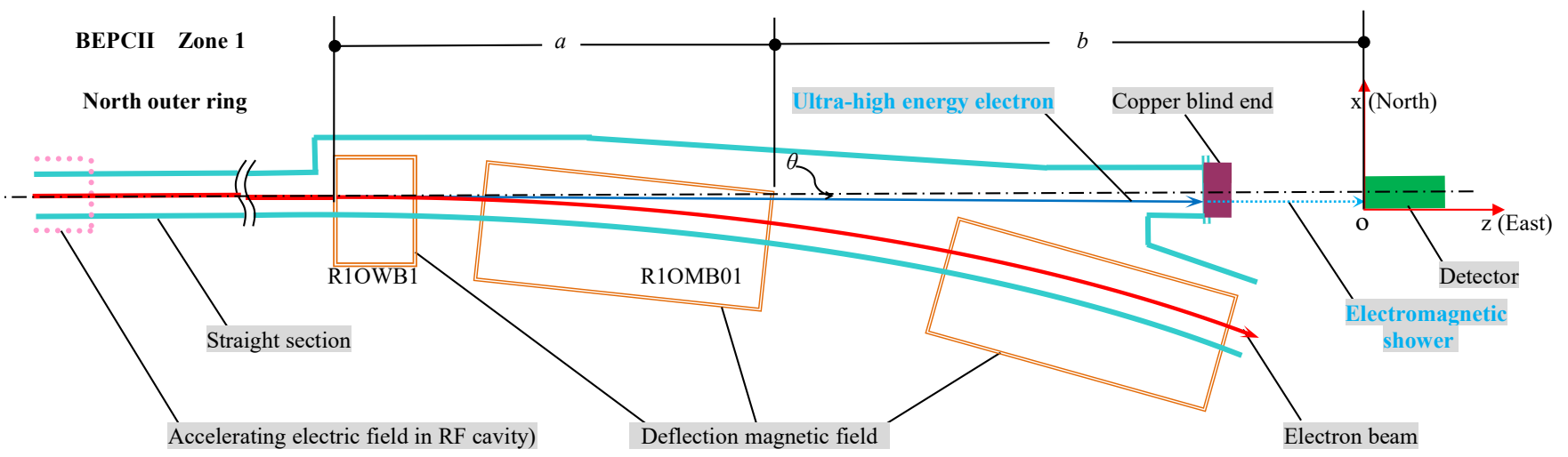

Figure 1. HSLM effect will cause the generation of abnormal ultra-high energy electrons with a certain small probability when the electron beam posses through the accelerating electric field

Relevant formulas are given below. For convenience, the energy $E_{R}{ }^{*}$ of ultra-high energy electron is expressed by its ratio $\varepsilon$ to the beam energy $E_{R}$,

$$
\varepsilon=E_{R}^{*} / E_{R}
$$

Since the fluctuation of beam energy $E_{R}$ is small and the focus of this research is $E_{R}{ }^{*} \gg E_{R}, E_{R}$ can 
be regarded as a constant. It can be proved that the occurrence rate (unit: $\mathrm{s}^{-1}$ ) of ultra-high energy electrons with a certain energy $E_{R}{ }^{*}$, i.e. certain $\varepsilon$ is

$$
f_{\varepsilon}=\frac{k I U \sin \omega}{\varepsilon-1}
$$

$U$ is the RF voltage (unit: $\mathrm{V}$ ), $\omega$ is the phase angle (unit: ${ }^{\circ}$ ), $I$ is the average beam current (unit: A), and $k$ is a constant

$$
k=\frac{4}{3 \Phi E_{0}}=1.57 \times 10^{-6}\left(\mathrm{~J}^{-1}\right)
$$

The highest energy of ultra-high energy electrons occurring during a enough long time $T$ (unit: s)

$$
\varepsilon_{\max }=k I U T \sin \omega+1
$$

The number of ultra-high energy electrons between a certain $\varepsilon$ to $\varepsilon_{\max }$ is

$$
n=k I U T \sin \omega \cdot \ln \frac{k I U T \sin \omega}{\varepsilon-1}
$$

In addition, due to the action of the deflection magnetic field, the ultra-high energy electron will deviate from its previous track direction by a small angle $\theta$ (see Fig.1),

$$
\theta \approx \frac{a(a+2 b)}{2 r(a+b) \varepsilon} \quad(\varepsilon>>1)
$$

On the BEPCII, $a=2.37 \mathrm{~m}$ and $b=4.66 \mathrm{~m}$, the equivalent deflection radius of the combination of deflection magnets R1OWB1 and R1OMB01 is $r=16.38 \mathrm{~m}$. For the ultra-high energy electron with the highest energy that appears in a long enough time, such as one with $\varepsilon_{\max }=2.15 \times 10^{5}$, calculated $\theta_{\text {mix }} \approx 0.1^{\prime \prime}$, which can be regarded as keeping the original direction in the measurement error. For electromagnetic showers in the copper blind end caused by ultra-high energy electrons respectively with energy of $\varepsilon_{\max }$ and $\varepsilon<\varepsilon_{\max }$, there is a distance $\delta$ between two dropping points of their axis lines on the detector,

$$
\delta \approx \frac{a(a+2 b)}{2 r \varepsilon}=\frac{\rho}{\varepsilon} \quad(\varepsilon>>1)
$$

Taking the experiment of $\$ 3.3 .2$ as an example, two important features of ultra-high energy electrons are expected by calculating: (1) The abnormal electron with the highest energy $E_{R}{ }^{*}(\max ) \approx 433 \mathrm{TeV}$ can be generated. (2) If the lower limit of energy of electrons escaping from the storage ring is set at $\left(1+10^{-2}\right) \cdot E_{R}$, the number of electrons lost due to the HSLM effect is $n<6 \times 10^{5}$ during $77 \mathrm{hrs}$ operation. In that run, BEPCII performed a beam injection approximately every 1 hour and before each injection the beam current has decreased from $\sim 500 \mathrm{~mA}$ to $\sim 400 \mathrm{~mA}$, so more than $10^{13}$ electrons were lost in 77 hours, which is much greater than the loss from the HSLM effect. The impact of HSLM effect is so weak, no wonder people have never noticed it before.

\subsection{Experimental method}

\subsubsection{The calorimeter for detecting electromagnetic shower and its placement}

As mentioned in the experiment principle of $\$ 3.1$, to search for ultra-high energy electrons on BEPCII electron storage ring is achieved by detecting the electromagnetic shower coming from the copper blind end of the downstream of RF cavity, so a sampling electromagnetic calorimeter can be used as a detector, and the copper blind end at this time equals to the front end absorber of calorimeter. Fig.2 is the diagram of the experimental setup on the BEPCII, due to environmental constraints, the author's detector was placed $1.53 \mathrm{~m}$ away from the copper blind end, of course, it is ideal to place the detector close to the copper blind end. 


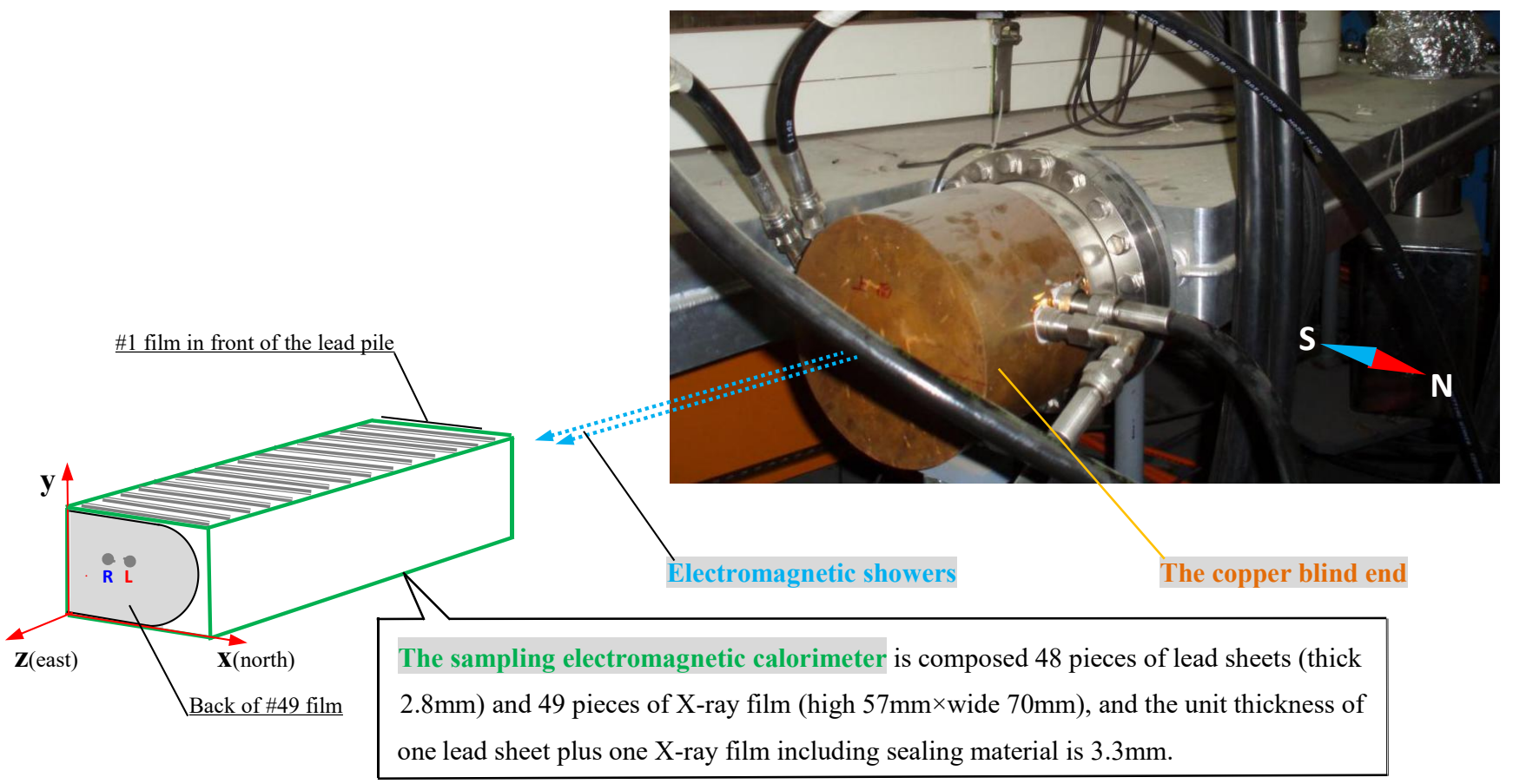

Figure 2. The copper blind end of downstream of the north outer straight section of zone 1 of BEPCII electron storage ring and the sampling electromagnetic calorimeter

\subsubsection{About the background and the detection of single event}

The main interference to the detection of ultra-high energy electrons is the bremsstrahlung (hereinafter referred to as GB) caused by the interaction between the electron beam and the residual gas in the vacuum chamber, which is much strong than X-ray from the RF cavity and synchrotron radiation ${ }^{[2][3]}$. Therefore, the following will directly use GB as the background.

Although the total intensity of GB is high, the shower particles number maximum $N_{\max (\mathrm{GB})}$ and position maximum $t_{\max (\mathrm{GB})}$ caused by one photon are much low than the $N^{*}{ }_{\max }$ and $t^{*}{ }_{\text {max }}$ caused by one ultra-high energy electron. The reference[4] gives formulas calculating for shower electrons:

$$
\begin{aligned}
& N_{\max }=\frac{0.3 E}{E_{c} \sqrt{\ln \frac{E}{E_{c}}-\mathrm{A}}},\quad \text { (for photon } \mathrm{A}=0.18, \text { for electron } \mathrm{A}=0.37) \\
& t_{\max }=\ln \frac{E}{E_{c}}+\mathrm{B}, \quad(\text { for photon } \mathrm{B}=0.5, \text { for electron } \mathrm{B}=-0.5)
\end{aligned}
$$

For example, on BEPCII, it is calculated by Eq.(14) that the maximum of shower electrons number in lead caused by one ultra-high energy electron of $E_{R}{ }^{*} \geq 3.4 \mathrm{TeV}$ (that corresponds to $\delta \leq 0.5 \mathrm{~mm}$ ) is $N_{\text {max }}^{*}>10^{4}$; while according to Fig. 8 , the maximum number of shower particles caused by GB in 77 hours is $3 \times 10^{11}$, which is equivalent to one shower particles per a microsecond. Also it is be calculated by Eq.(15) that the lead thickness of the shower maximum from the ultra-high energy electrons are much bigger that from GB, that is, $\Delta t=t^{*}{ }_{\max }-t_{\max (\mathrm{GB})}>6.4 X_{0}>36 \mathrm{~mm}$. Therefore, if a lead plate of a certain thickness is added in front of the detector, and the window time of the detector is appropriately set, the background can be effectively filtered out.

In short, using the electromagnetic calorimeter with an online real-time display function, such as the "Shashlik Calorimeter", a single ultra-high energy electronic signal can be effectively identified in the background radiation. When enough events are obtained, a quantitative verification to the 
theory can be done by comparing with the results calculated by formulas (7) (13).

\subsubsection{The detection of the accumulation of a large number of events}

In addition to use the method of detection to a single event, the existence of ultra-high energy electrons can be tested by detecting the cumulative result of a large number of events during a long time. This article reports the experiment detecting cumulative effect.

The rationality and feasibility of this detecting method can be illustrated by the Monte Carlo simulation. Fig. 4 and Fig. 8 are the simulation results using FLUKA program: The red and orange curves are the simulation of the longitudinal distribution of accumulated electromagnetic showers in detector lead caused by the GB which is as main component of the background, and the blue curves are the simulation of the longitudinal distribution of accumulated electromagnetic showers in detector lead caused by the ultra-high energy electrons which are calculated by the formula (11).

The simulation curves show: (1) If there are no the ultra-high energy electrons, the accumulated electromagnetic shower caused by the background radiation with GB as the main component is very strong in the early stage of development, but it attenuates rapidly and proportionally after $\mathrm{Pb}$ thickness $\sim 70 \mathrm{~mm}$; (2) The accumulated electromagnetic shower caused by the ultra-high energy electrons much weaker than the shower caused by GB in the early stage of development, but after $\mathrm{Pb}$ thickness $\sim 70 \mathrm{~mm}$ it will gradually approach and eventually exceed the shower caused by GB.

Therefore, if ultra-high energy electrons do exist, after enough deep lead layer, the development of shower will appear abnormal phenomena different to the normal background attenuation, so the qualitative and semi-quantitative verification to ultra-high energy electrons can be achieved by detecting the abnormal attenuation phenomena consistent with theoretical prediction.

Based on the above analysis, the author used the sampling calorimeter shown in Fig.2 to detect the cumulative electromagnetic shower coming from the copper blind end of downstream of straight section of north outer ring of zone 1 of BEPCII electron storage ring. The detector is composed by 48 sheets of lead with a thickness of $2.8 \mathrm{~mm}$ and 49 sheet of X-ray films. During this period run of BEPCII, the series of X-ray films is in continuous exposure, thereby recorded the distribution of cumulative shower particles in lead at intervals of $1 / 2$ radiation length.

Developed films are input to the computer via the scanner, then to observe the photosensitive spots on film under the brightness and contrast appropriate adjusted by WPS software. It is important to look closely those photosensitive spots on films after the $\mathrm{Pb}$ depth $70 \mathrm{~mm}$ (\#26 film), first identify out a set of photosensitive spots of pure background, through comparing with it to look if there are abnormal photosensitive spots that match the theoretical simulation.

\subsection{Experimental results}

The author did first experiments at NSRL, the signs of ultra-high energy electrons are found ${ }^{[5]}$. Next at the more suitable BEPCII, two long time accumulating detection under different conditions were performed, as will be stated in detail below, the abnormal phenomena caused by theoretically expected ultra-high energy electrons was all detected in both experiments.

\subsubsection{Detection of ultra-high energy electrons emission during BEPCII single-ring operation}

When BEPCII is in working the single-ring, the electron beam flows from the north outer ring of Zone 2 into the north outer ring of Zone 1 via a short straight section $\underline{C D}$, the layout is shown in Figure 5. During the experiment, $E_{R}=2.5 \mathrm{GeV}, I=185 \mathrm{~mA}, U \sin \omega \approx 1.3 \mathrm{MV}$. During 233 hours run, the beam orbit was stable, its XPOS float $<0.1 \mathrm{~mm}$. Using formulas (7) (13), the feature values of ultra-high energy electrons are calculated (See Tab.1), and the longitudinal development of 
electromagnetic shower in detector lead caused by the GB and ultra-high energy electrons within $791 \mathrm{TeV} \geq E_{R}{ }^{*} \geq 4.25 \mathrm{TeV}$ are simulated respectively (See Fig.4). For the experimental results, first give general explanation, and finally to display and analysis the films taken in detail.
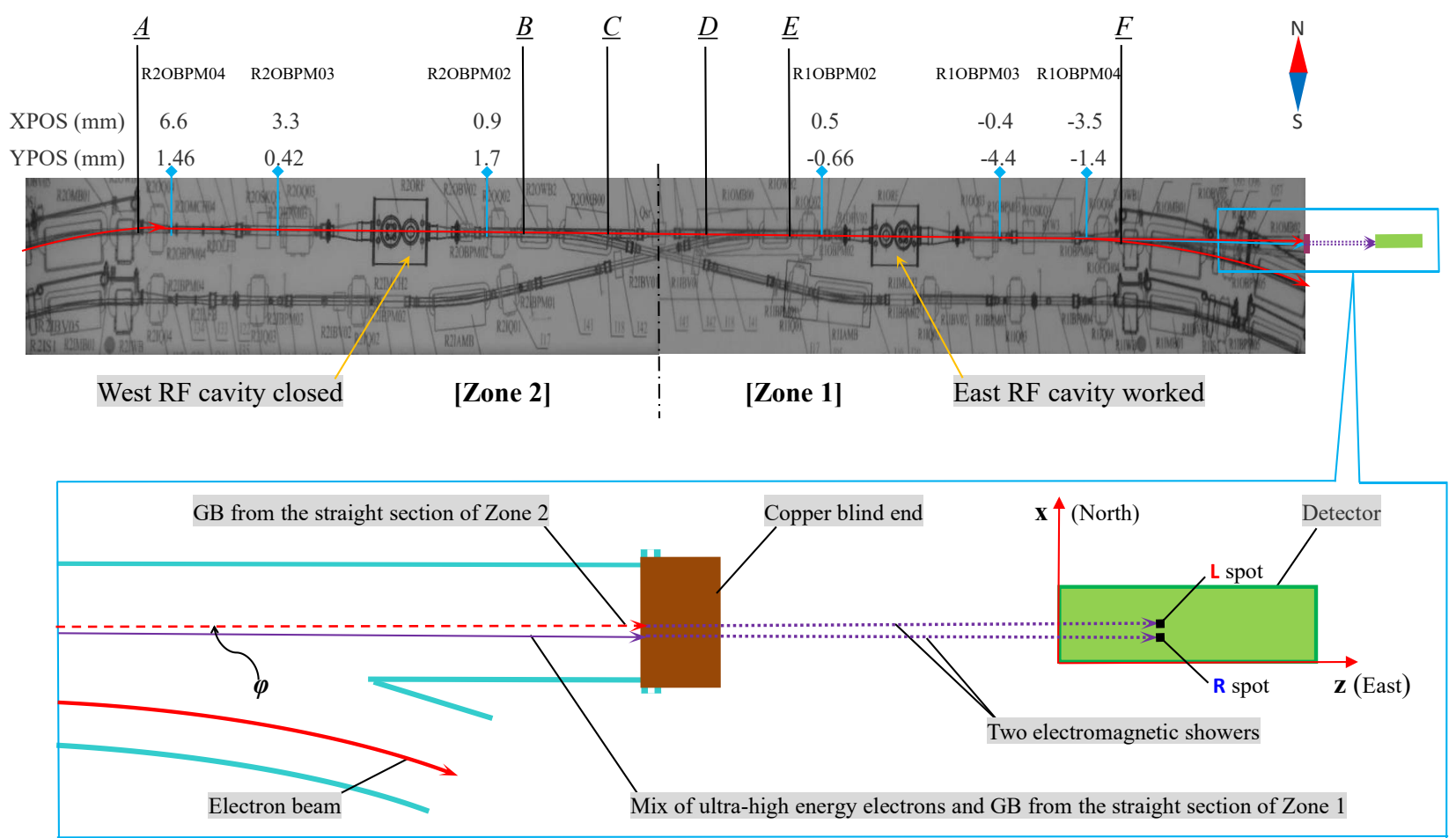

Figure 3. The gas bremsstrahlung (GB) and ultra-high energy electrons cause electromagnetic showers in the copper blind end of downstream of the east RF cavity of the electronic storage ring of BEPCII

Table 1. Calculated values of ultra-high energy electrons under $E_{R}=2.5 \mathrm{GeV}, I=185 \mathrm{~mA}, U \sin \omega \approx 1.3 \mathrm{MV}, T=233 \mathrm{hrs}$

\begin{tabular}{|c|c|c|c|c|}
\hline \multicolumn{2}{|c|}{ Maximum of energy } & Number & \multicolumn{2}{c|}{ Start to exceed the background shower } \\
\hline$E_{R}{ }^{*} \max$ & $\varepsilon_{\max }$ & $E_{R}{ }^{*} \geq 4.25 \mathrm{TeV} \quad($ i.e. $\delta \leq 0.5 \mathrm{~mm})$ & Lead depth $(\mathrm{z})$ & Matching film \\
\hline $791 \mathrm{TeV}$ & $3.16 \times 10^{5}$ & $1.65 \times 10^{6}$ & $76 \mathrm{~mm}$ & Between \#28 and \#29 \\
\hline
\end{tabular}

\section{(i) About the normal electromagnetic shower caused by the residual gas bremsstrahlung}

The electromagnetic shower in detector lead caused by the GB in the long straight section of north outer ring of BEPCII constitutes the main component of background. It is well known that the electromagnetic shower has a "core" ${ }^{[4]}$, but on films before \#30 film (Pb thickness $\left.81.2 \mathrm{~mm}\right)$ the shower core is submerged in the photosensitive area formed by a large number of secondary particles, so that it was not visible. After the shower develops to \#30 film, the core gradually appeared, and it is surprising that the core is not one, but two, they are about $10 \mathrm{~mm}$ apart, that are $\mathrm{L}$ spot and R spot (See \#30 to \#49). In this experiment, the existence of two core spots $L$ and $R$ of electromagnetic shower is interest, which provides the following information:

(1) When the single-ring run, although four magnets R2OWB2, R2OMB00, R1OMB00, R1OWB2 that only used during double-ring run have turned off, there is still a very weak remanence. Under the action of this residual weak magnetic field, the electron beam that flows from the straight section $\underline{A B}$ to straight section $\underline{E F}$ forms a small deflection toward the center (south) of the storage ring, thereby form two strand of the GB in the straight sections $\underline{A B}$ and $\underline{E F}$, and which further cause two strand of electromagnetic showers in the copper-lead, the blackest spots $L$ 
and $\mathrm{R}$ are their respective cores. Based on the distance of $\sim 10 \mathrm{~mm}$ of $L$ and $R$ and the geometric size of storage ring, it can be calculated that the deflection angle of the beam from section $\underline{A B}$ to $\underline{E F}$ is $\varphi \approx 1^{\prime} 24^{\prime \prime}$, and the overall average intensity of remanence is about 8 Gauss. By the Gauss meter to detect, actual intensities of four magnets are 5.3, 10.9, 10.4 and 7.9 Gauss in sequence, the calculation is consistent with the fact, and so this consistency also has supported the above analysis.

(2) The blackest spots $L$ and $R$ reflect the existence of two cores of strong electromagnetic shower, which shows that although the electron beam flow is oscillating, the GB formed in the straight section without the dipole magnet is like a linear emission with a high-density core. However, the photosensitive spots distributed between $L$ and $R$ are weak and uniform, which shows that the electron beam in sections $\underline{B C}$ and $\underline{D E}$ covered by the weak magnetic field moves in an arc, so as to form the scattered GB and cause the weak and uniform electromagnetic shower in detector lead.

(ii) There was a "pure" background without ultra-high energy components as the contrast

According to the author's theory, the ultra-high energy electrons will be generated when the electron beam passes through an accelerated electric field. During this experiment, only the RF accelerated cavity of Zone 1 was working, so when the electron beam passes through the section $\underline{E F}$, ultra-high energy electrons can be generated. However, there is no accelerated electric field in Zone 2, so the radiation from section $\underline{A B}$ is a "pure background" without ultra-high energy electrons, by which caused the photosensitive spot $L$ of shower appears on the left side of every film.

Under the action of the downstream deflection magnetic fields R1OWB1 and R1OMB01, the ultra-high energy electron will deviate from its previous motion direction according to Eq.(12) and (13). The calculation shows what can make a significant contribution to the cumulative results in the late stage of the development of electromagnetic shower are only ultra-high energy electrons of $\delta<0.5 \mathrm{~mm}$. The deviation $\delta$ is so small, it is impossible to distinguish out cores of which between the electromagnetic showers caused respectively by the GB and ultra-high energy electrons, the spot $R$ is actually a superposition of them. Due to the electron beam undergoes a small deflection toward the center of the storage ring from Zone 2 to Zone 1, the mixed spot $\mathrm{R}$ appears at the right of the pure background spot $L$ on films, and the distance between them is about $10 \mathrm{~mm}$.

In short, precisely because the deflection of small angle of the electron beam from Zone 2 to Zone 1 and the alone work of RF cavity of Zone 1, so there is a pure background as a contrast in this experiment, which can help us to find the expected ultra-high energy signals in the mixed photosensitive spots.

(iii) To find the expected ultra-high energy signals by contrasting with the pure background

(1) To find the expected signals by comparison between the spots $L$ and $R$ on a same film

Because the intensity of GB relates the pressure of residual gas, and the gas pressure of different places of the storage ring have usually some difference, so the intensity of electromagnetic shower caused by GB of sections $\underline{A B}$ and $\underline{E F}$ will be different. From photosensitive spots in the early stage of development of shower, it can be seen that $\mathrm{L}$ spots originating from the section $\underline{A B}$ are larger and darker than $\mathrm{R}$ spots originating from the section $\underline{E F}$, which shows that the gas pressure of the section $\underline{A B}$ is higher than the gas pressure of the section $\underline{E F}$. Based on this, two curves of electromagnetic shower caused by GB are simulated, the red curve related the section $\underline{A B}$ with higher gas pressure and the orange curve related the section $\underline{E F}$ with lower gas pressure (See Fig.4).

According to these two curves, if there only are the GB and other known low energy radiations in the storage ring, the $L$ spot should be always larger than $R$ spot on each film, but the detected results are not the case. In fact, on some films from \#29 to \#49, the size of photosensitive spot is no 
longer $L>R$ but $R>L$, especially on $\# 29$ film (Pb depth $78.4 \mathrm{~mm}$ ) it is $R>>L$. After $\# 39$ it is all $R>L$. This shows that there are ultra-high energy components in radiations of the $\underline{E F}$ section having the accelerating electric field, which does large contribution in the later stage of the development of electromagnetic shower.

The bright blue curve in Fig. 4 is the total of two simulated curves of the electromagnetic shower caused by the ultra-high energy electrons (gray blue) and the GB (orange) in section $\underline{E F}$. At $\mathrm{Pb}$ thickness $\sim 76 \mathrm{~mm}$ (between \#28 and \#29) the bright blue curve intersects with the red curve, and at $\mathrm{Pb}$ thickness is larger than $\sim 76 \mathrm{~mm}$ the bright blue curve is higher than the red curve. On \#29 film at $\mathrm{Pb}$ thickness $78.4 \mathrm{~mm}$, it can be seen that the size of spot $\mathrm{R}$ is much large than spot $\mathrm{L}$, which indicates that the theoretical expectation is consistent with the measured result.

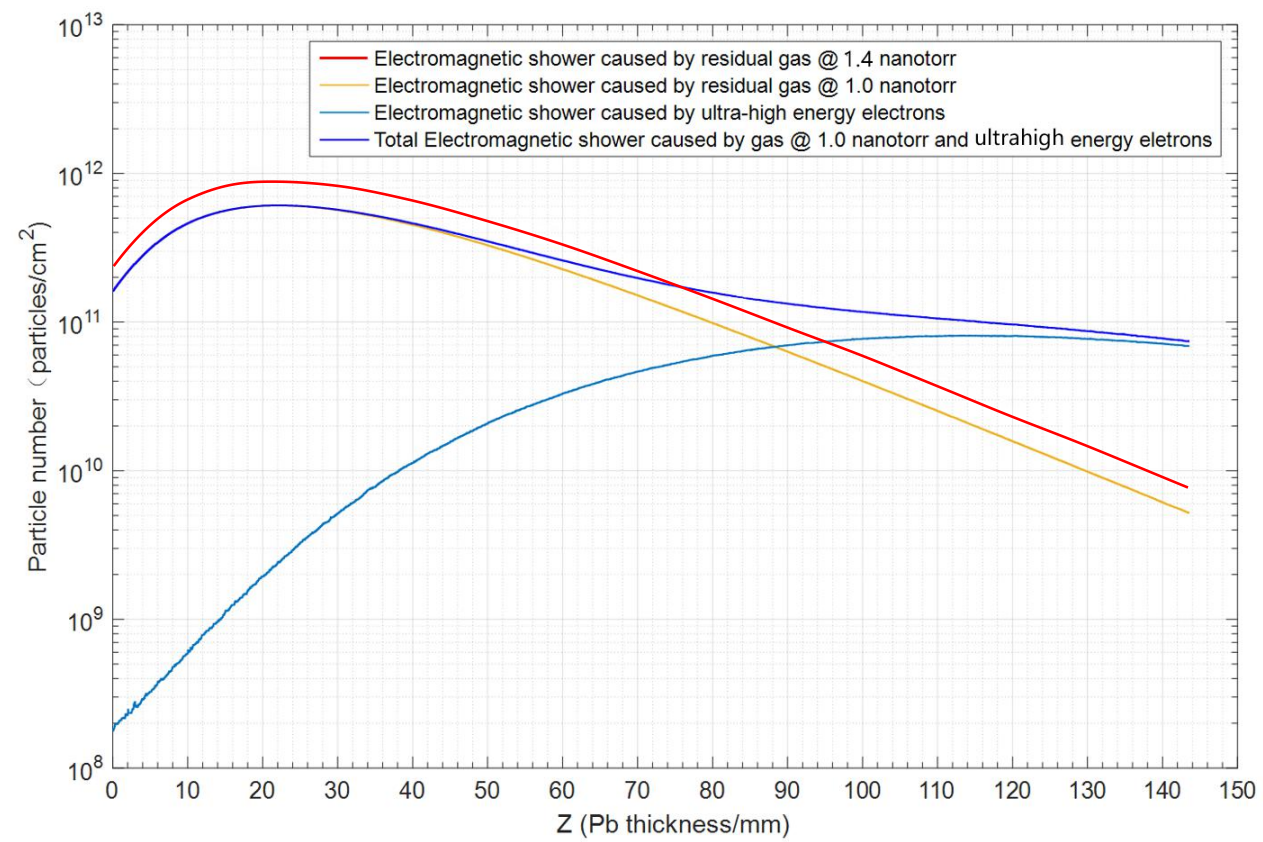

Figure 4. Simulation of the longitudinal distribution of the electromagnetic showers in the detector lead caused by three strands of radiation from two long straight section of the north outer ring of BEPCII

\section{(2) To find expected signals by comparison of the attenuation feature of series of $L$ with $R$}

In a cumulative detection of up to ten days, if two series of photosensitive spots $L$ and $R$ are all formed by the same known effect, then their law of attenuation is certainly the same. However, the actual detection shows that their attenuation characteristics are not same: The series of spot $L$ of pure background attenuates rapidly in proportional, but the attenuation of the series of spot $\mathrm{R}$ that the background mixes with ultra-high energy electrons is slow and ups and downs.

This measured result is also in line with theoretical prediction, it can be seen in Fig.4 that the bright blue curve simulating the mix of background with ultra-high energy electrons descends more slowly and more gently than the red curve simulating alone the background. In addition, because the occurrence of ultra-high energy electrons is after all random, it will inevitably bring ups and downs, and so to affect the distribution of electromagnetic shower in the deep of lead layer.

\section{(iv) To display and analyze films under different brightness (B) and contrast (C)}

The following to display and analyze the photosensitive spots of electromagnetic shower on films under different brightness (B) and contrast (C). Taking a group of 4 film to adjust B and C. The $\mathrm{B}$ and $\mathrm{C}$ of the original film are all $50 \%$. The sequence number of the film is shown in Fig. 2. 
The following 4 films at the deepest part of the lead layer of detector show that the R spots-series has an anomaly on the attenuation characteristic compared with the $L$ spots-series formed by the pure background, that is, the size and blackness of the normal $L$ spots-series attenuates rapidly in proportional, but the attenuation of abnormal $\mathrm{R}$ spot-series is slow and irregular. The theoretically predicted ultra-high energy electrons will cause this phenomenon occur in the R spots-series in the later stage of the development of electromagnetic shower.

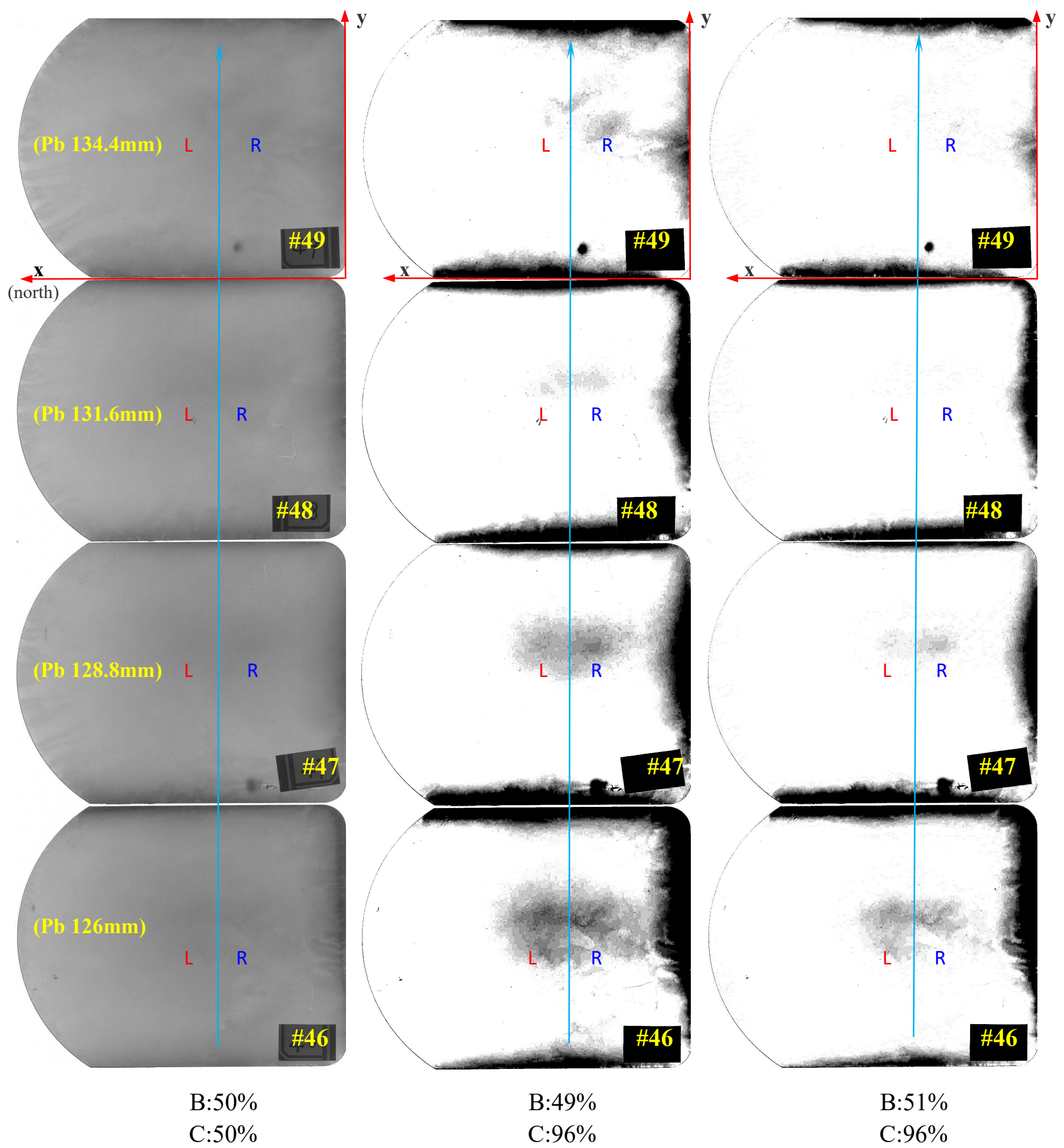


On films of the middle stage of the development of electromagnetic shower (See P15 and P16), it can be seen that the size of the $\mathrm{L}$ spots originating from the $\underline{A B}$ section are larger than the $\mathrm{R}$ spots from the $\underline{E F}$ section (which shows that the residual gas pressure in the $\underline{A B}$ section is slightly higher than the $\underline{E F}$ section), so if two strands of electromagnetic showers all are formed by the same known effects, the $L$ spot should always be larger than the R spot on a same film. However, the detection result is not the case. On this group of films in the later stages of the development of shower, the spot size on each film is not $L>R$ but $R>L$, which is exactly a phenomenon predicted by theory (See Fig.4).

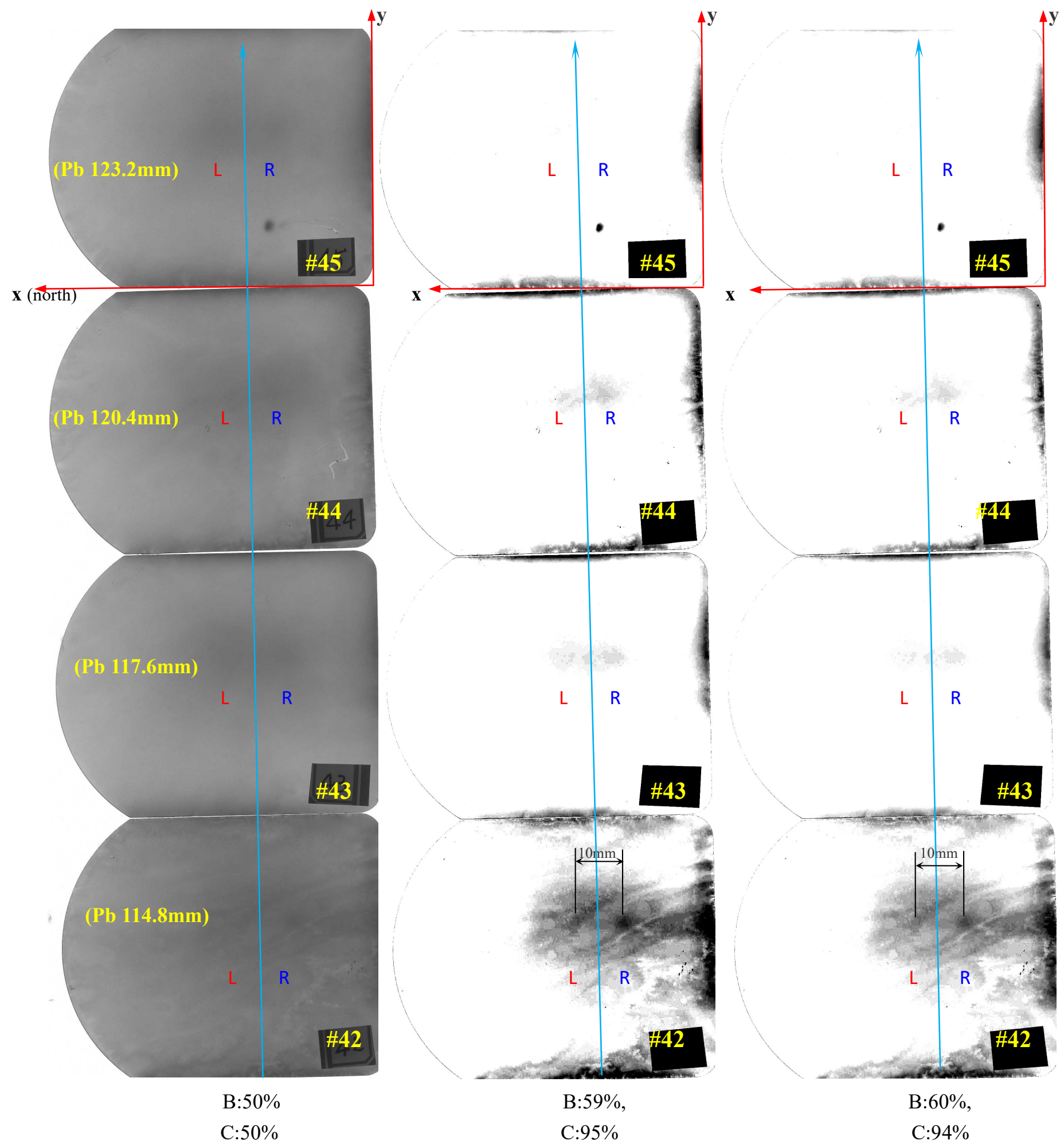


On this group of films, the normal $L$ spots-series originating from the pure background attenuates rapidly in proportional, but the abnormal $\mathrm{R}$ spots-series attenuates slowly and irregularly. In addition, after $\# 39$, the photosensitive spots on each film are all $\mathrm{R}>\mathrm{L}$. The detected phenomena are consistent with the theoretical prediction, it can be seen in Fig.4 that after Pb thickness $\sim 100 \mathrm{~mm}$ the purple curve simulating the sum of the ultra-high energy electrons and background descends more slowly.

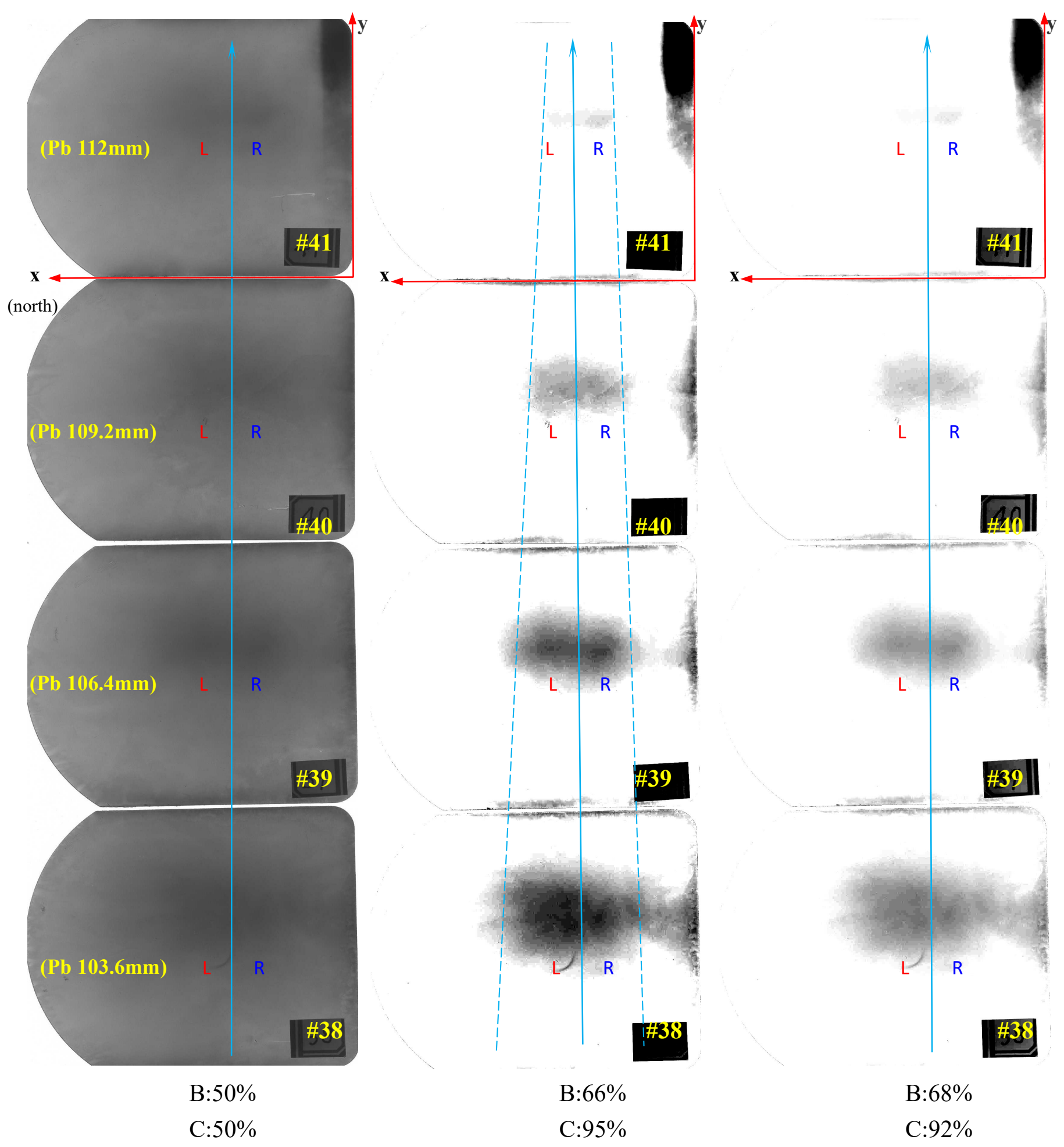


By comparison with the normal $L$ spots formed by the pure background radiation, it can be seen that the $\mathrm{R}$ spots are all abnormal on both features of "the attenuation of series" and "the comparison of left-right size on a same film", which is exactly the result predicted by the theory.

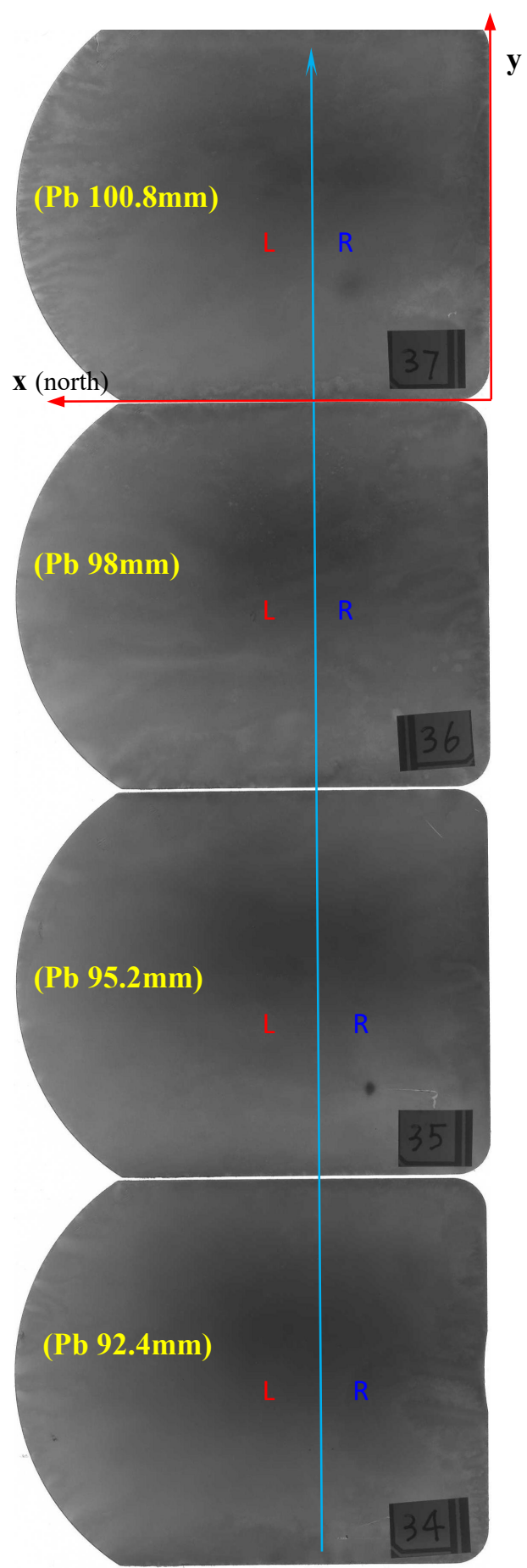

B: $50 \%$

C: $50 \%$

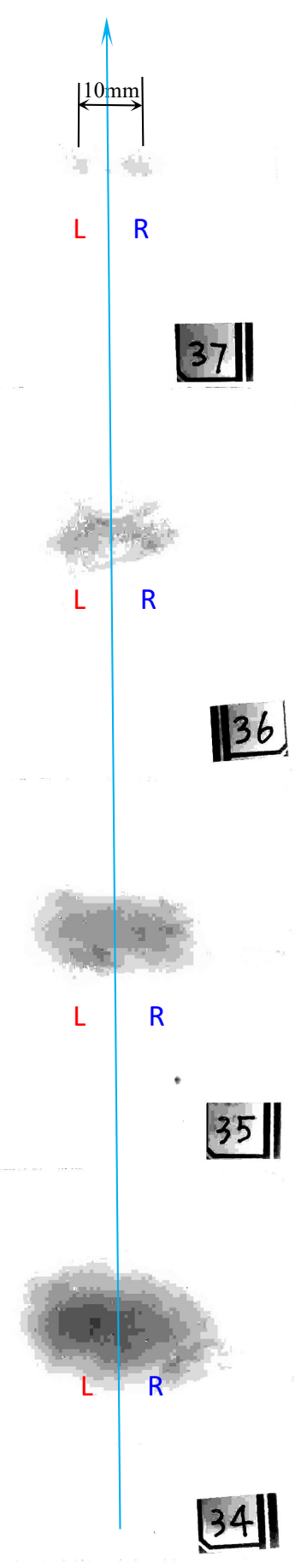

B: $77 \%$

$\mathrm{C}: 97 \%$

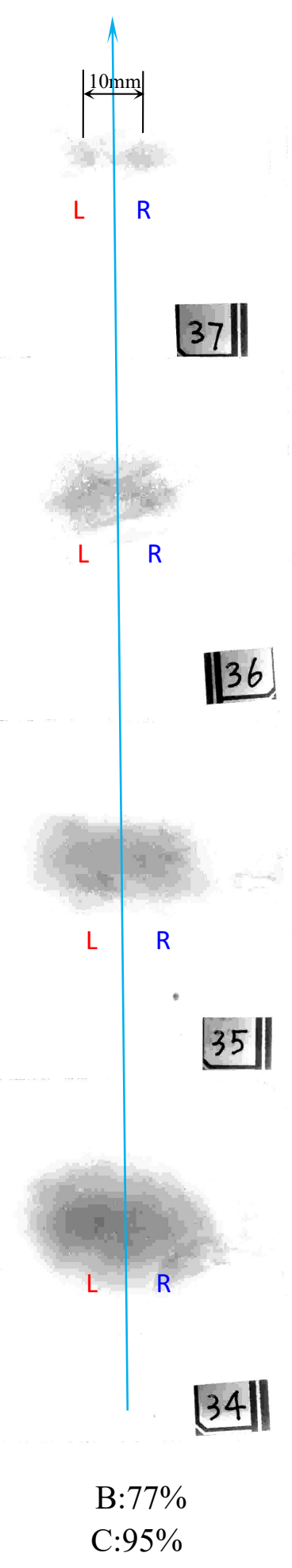


On this group of films, it can be seen that starting from the film \#30 two cores of two separated electromagnetic showers begin to emerge. Further seen that the abnormal attenuation feature of $\mathrm{R}$ spots-series is more clear compared to the L spots-series formed by the pure background, that is, the size and blackness of the $L$ spots attenuate rapidly and proportionally, while the attenuation of the $R$ spots are obvious slow, such as from \#30 film to \#31 film, the iso-blackness line of $L$ spot declines 2 layers but the R spot only declines 1 layer. Also, in the left-right spots comparison on \#31 film, the iso-blackness line of the $R$ spot is inversely higher one layer than the $L$ spot, which is not normal. The theoretically expected ultra-high energy electrons can cause these two abnormal phenomena.

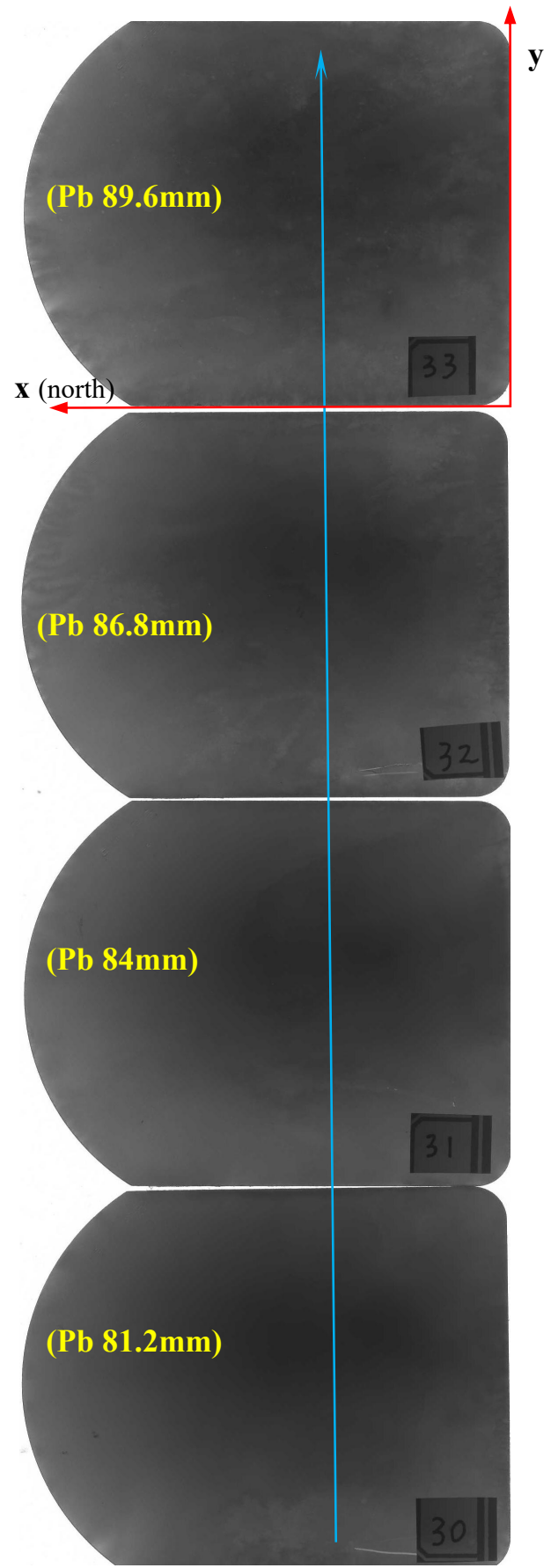

B: $50 \%$

C: $50 \%$

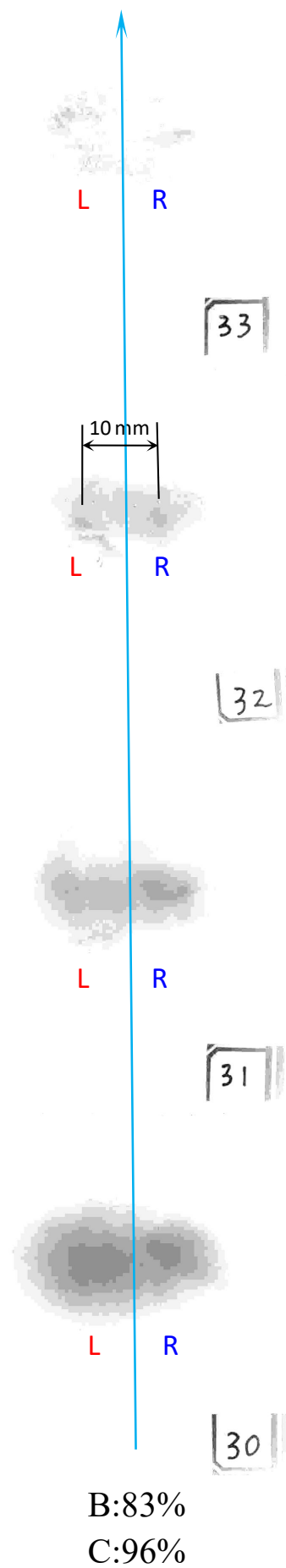

33

L

R

30

B: $83 \%$

C: $97 \%$ 
The darker photosensitive area on each film after \#26 (Pb thickness $70 \mathrm{~mm})$ becomes small and flattened from the previous approximate large round. Although the size of photosensitive area on films \#26 and \#27 still keep the left half larger than the right half, on the film \#28 the sizes of left and right have become roughly equal, while on the film \#29 (Pb thickness $78.4 \mathrm{~mm}$ ) the right half turns abnormally larger than the left half. This measured result is consistent with the theoretical predictions, it can be seen in Fig. 4 that starting from the Pb thickness $76 \mathrm{~mm}$ (between \#27 and \#28 film), the bright blue simulation curve excceds the red curve, that is, the electromagnetic shower caused by ultra-high energy electrons becomes stronger than the shower caused by GB.
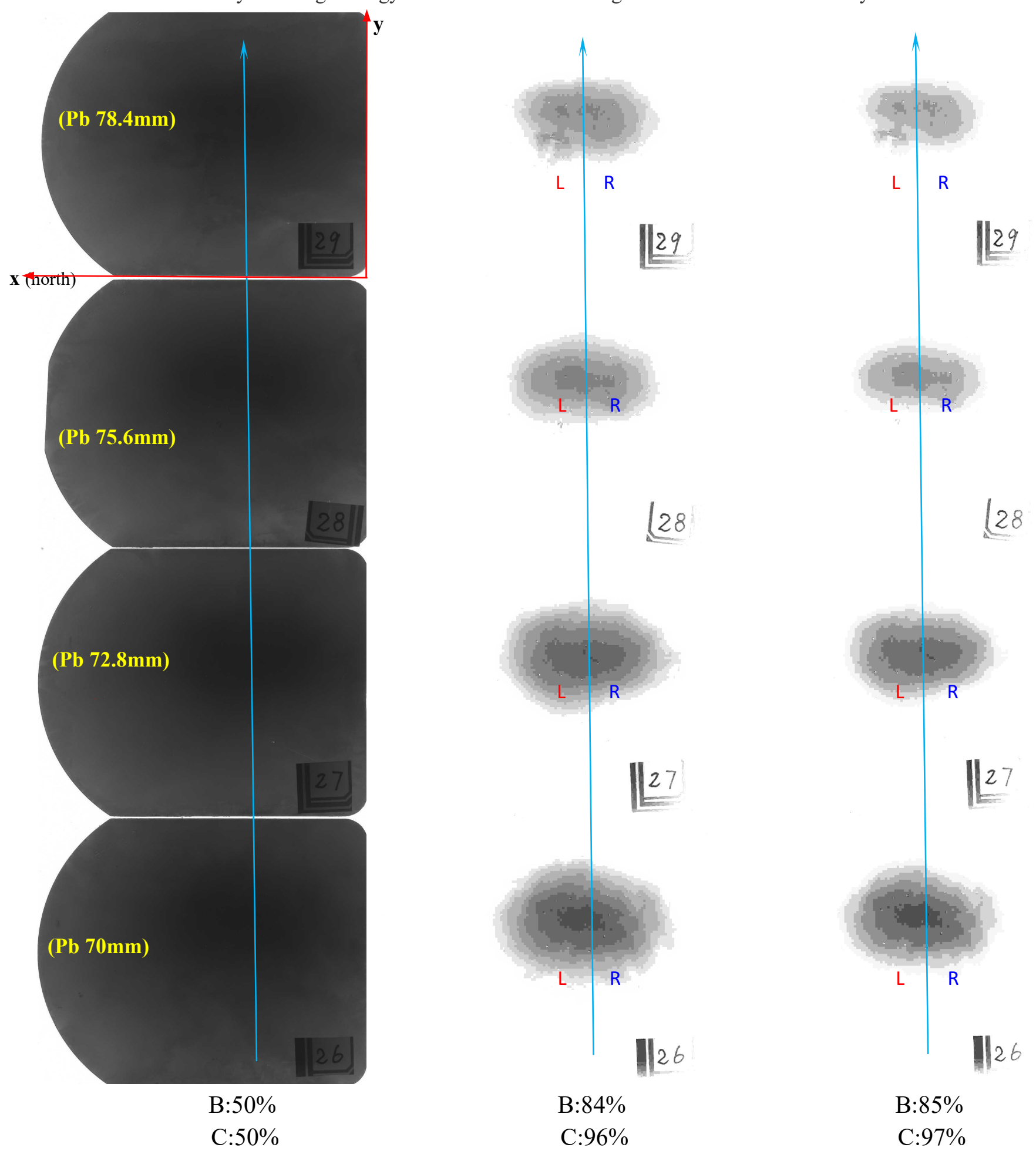
The photosensitive area on each film before \#25 is very large, this is a superposition result of a large number of secondary particles in scatter large angles caused by two strands of GB and low energy noise radiation. In the stage, the affect of ultra-high energy electrons is much lower than GB, which are consistent with the theoretical simulation (See Fig.4). Only the films under B: $86 \%$ and C: $97 \%$ are given here.
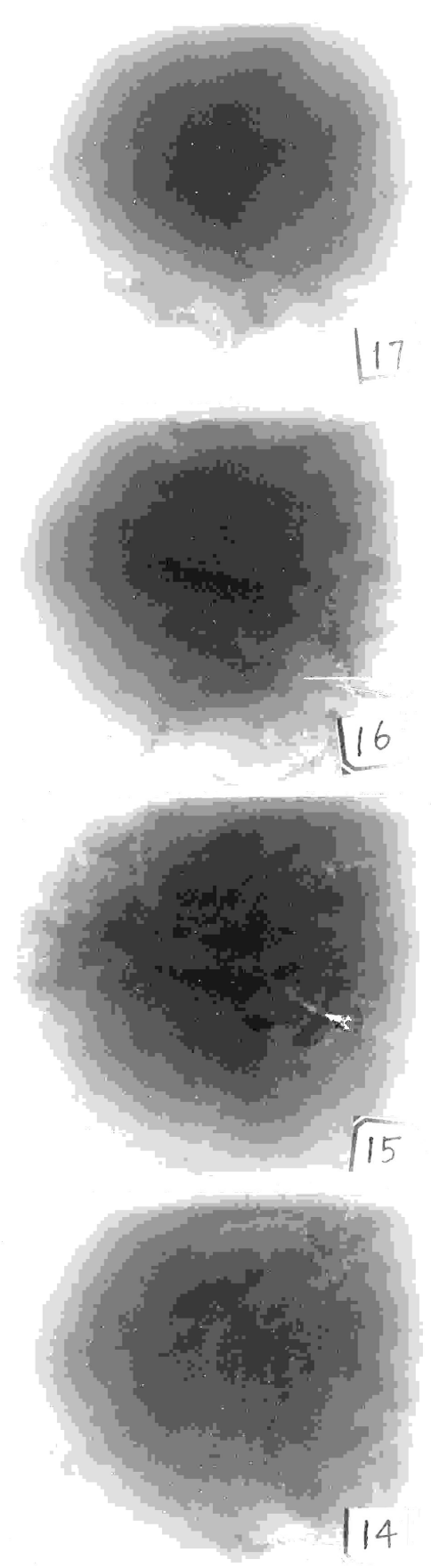
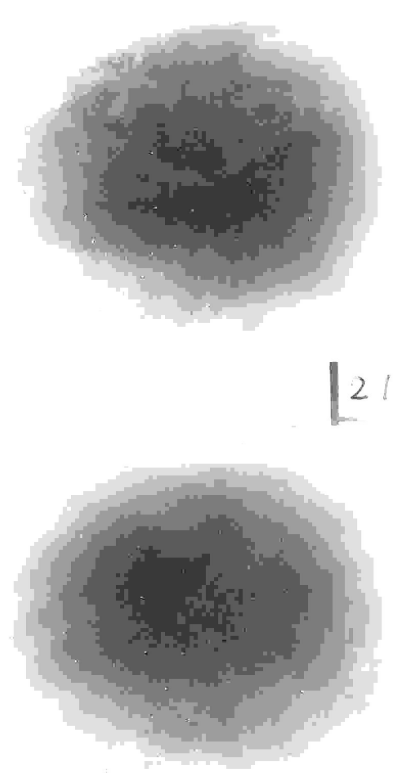

$\lfloor 20$
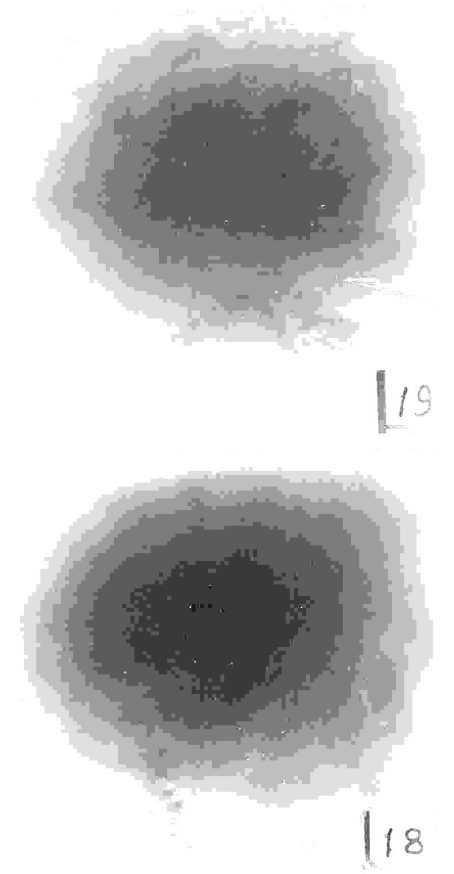
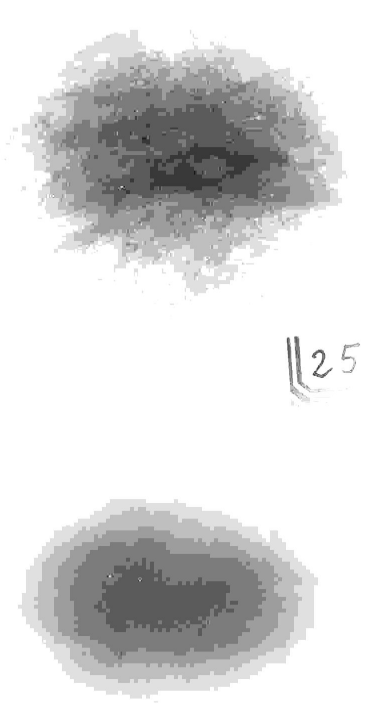

24

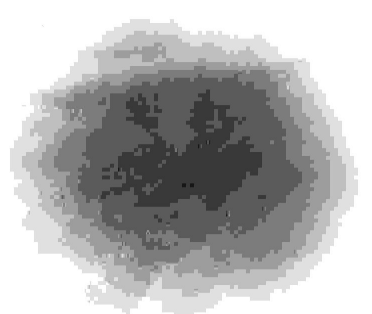

$\lfloor 23$

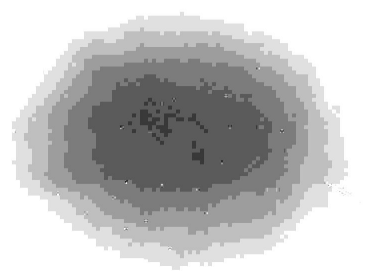

122 

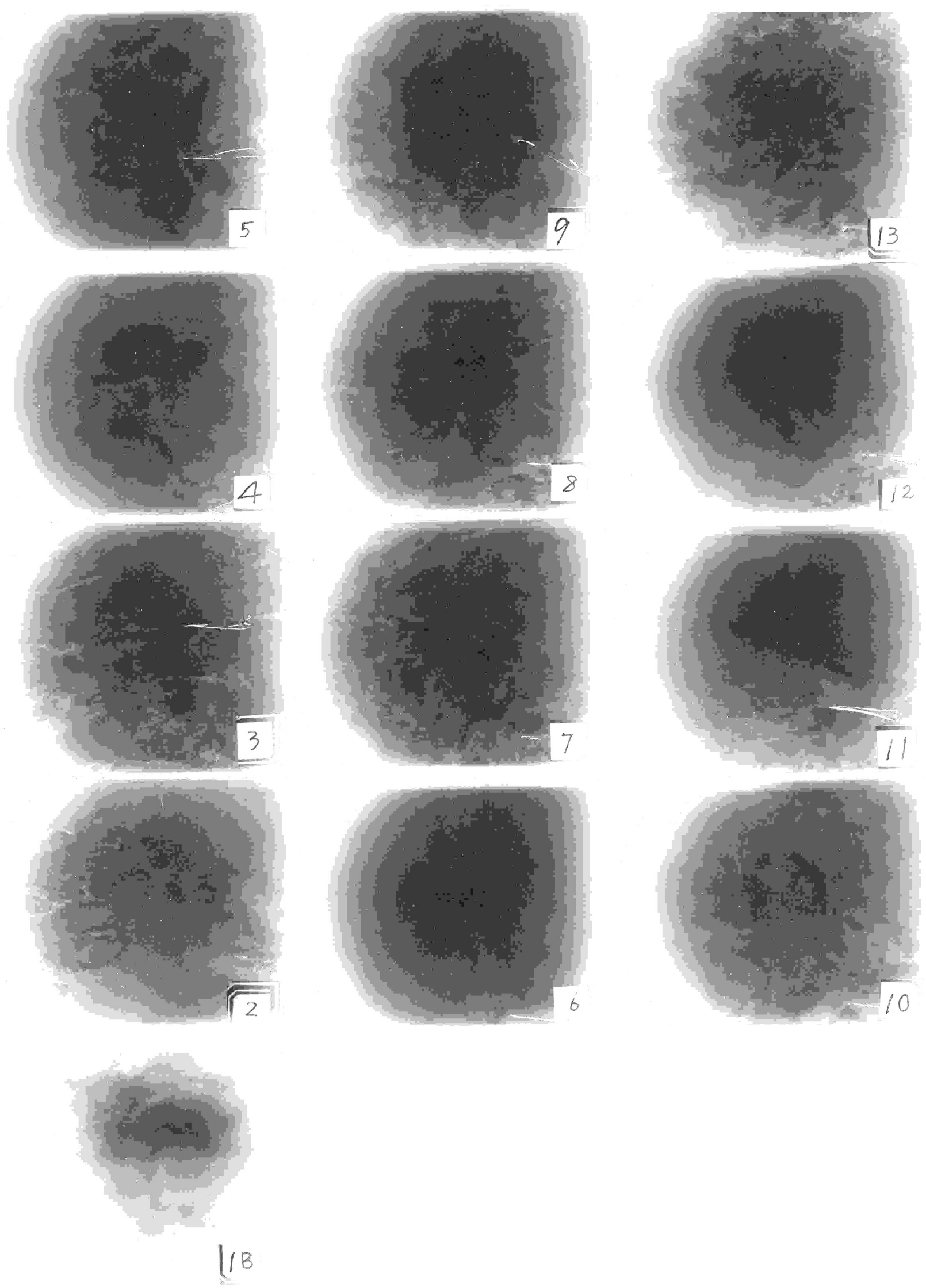


\subsubsection{Detection of ultra-high energy electron emissions during BEPCII double-rings operation}

In that experiment of the double-rings operation of BEPCII, $E_{R}=2 \mathrm{GeV}, I=450 \mathrm{~mA}, U=1.58 \mathrm{MV}$, $\omega=64.4^{\circ}$. The layout is shown in Figure 5.During this experiment, the electron beam steadily runs on the two orbitals for two time periods, which the position coordinate data and cumulative running time $T$ are written in the lower left of Fig.5. The theoretically calculated feature values of ultra-high energy electrons are listed in Tab.2. Figure 8 shows the simulation curves of the longitudinal development of the electromagnetic shower in the detector lead caused by the GB and ultra-high energy electrons within $433 \mathrm{TeV} \geq E_{R}^{*} \geq 3.4 \mathrm{TeV}$ are simulated respectively.

For the experimental results, first give general explanation, and finally to display and analysis the films taken in detail.
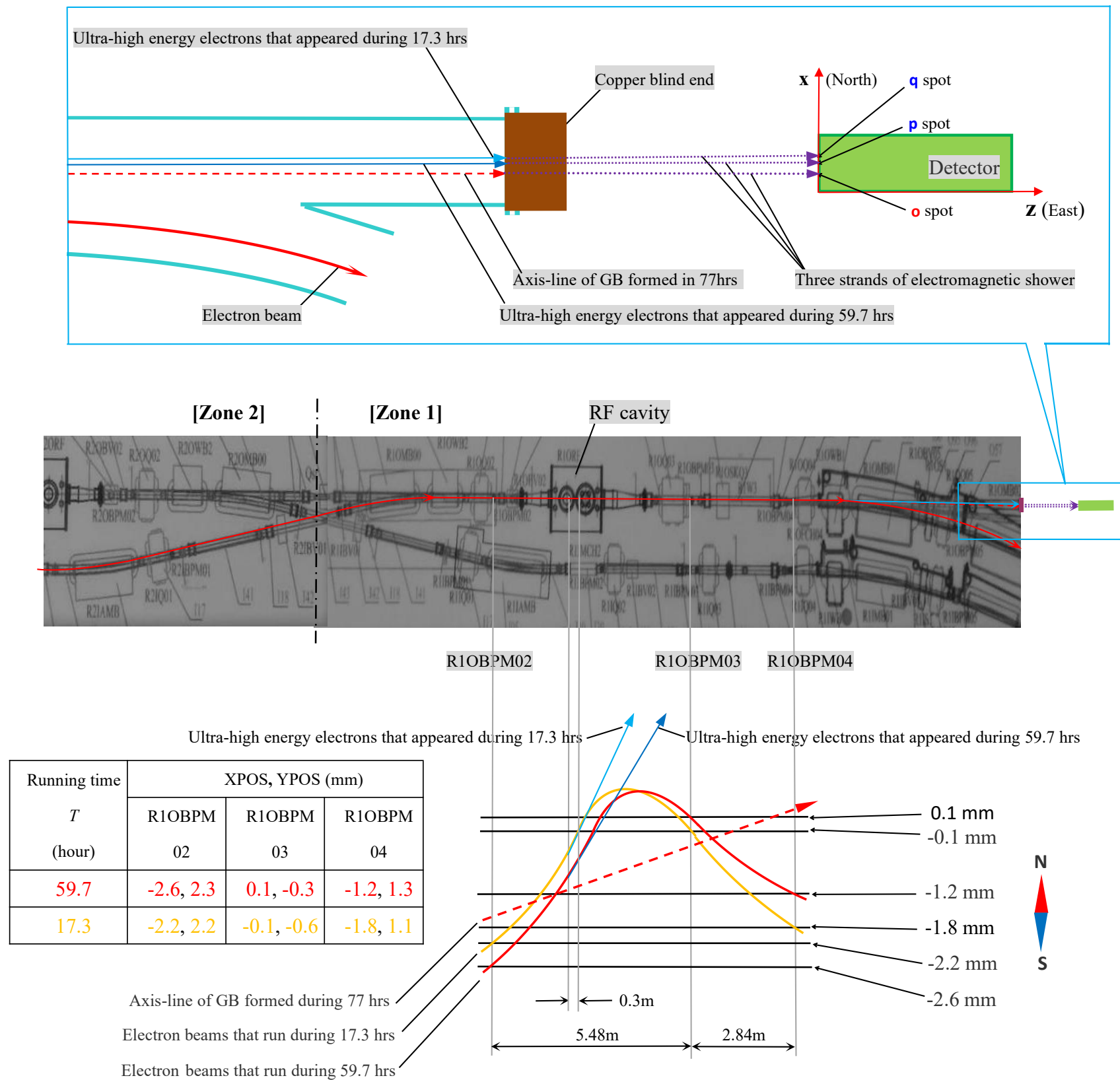

Figure 5. The operational parameters of electron beam and the enlarged schematic diagram of the emission direction of the ultra-high energy electrons and gas bremsstrahlung (GB) 
Table 2. Calculated values of ultra-high energy electrons under $E_{R}=2 \mathrm{GeV}, I=450 \mathrm{~mA}, U=1.58 \mathrm{MV}, \omega=64.4^{\circ}$

\begin{tabular}{|c|c|c|c|c|}
\hline \multicolumn{2}{|c|}{ Maximum of energy } & \multicolumn{2}{c|}{ Number } & \multicolumn{2}{c|}{ Start to exceed the background shower } \\
\hline$E_{R}{ }^{*}$ max & $\varepsilon_{\max }$ & $E_{R}{ }^{*} \geq 3.4 \mathrm{TeV}$ (i.e. $\left.\delta \leq 0.5 \mathrm{~mm}\right)$ & Lead depth $(\mathrm{z})$ & Matching film \\
\hline 433 & $2.16 \times 10^{5}$ & $1.05 \times 10^{6}$ & 87 & Between \#32 and \#33 \\
\hline
\end{tabular}

(i) First to watch the situation of the \#1 film placed on front of the lead pile

The \#1 film under the different brightness and contrast is displayed as follows:
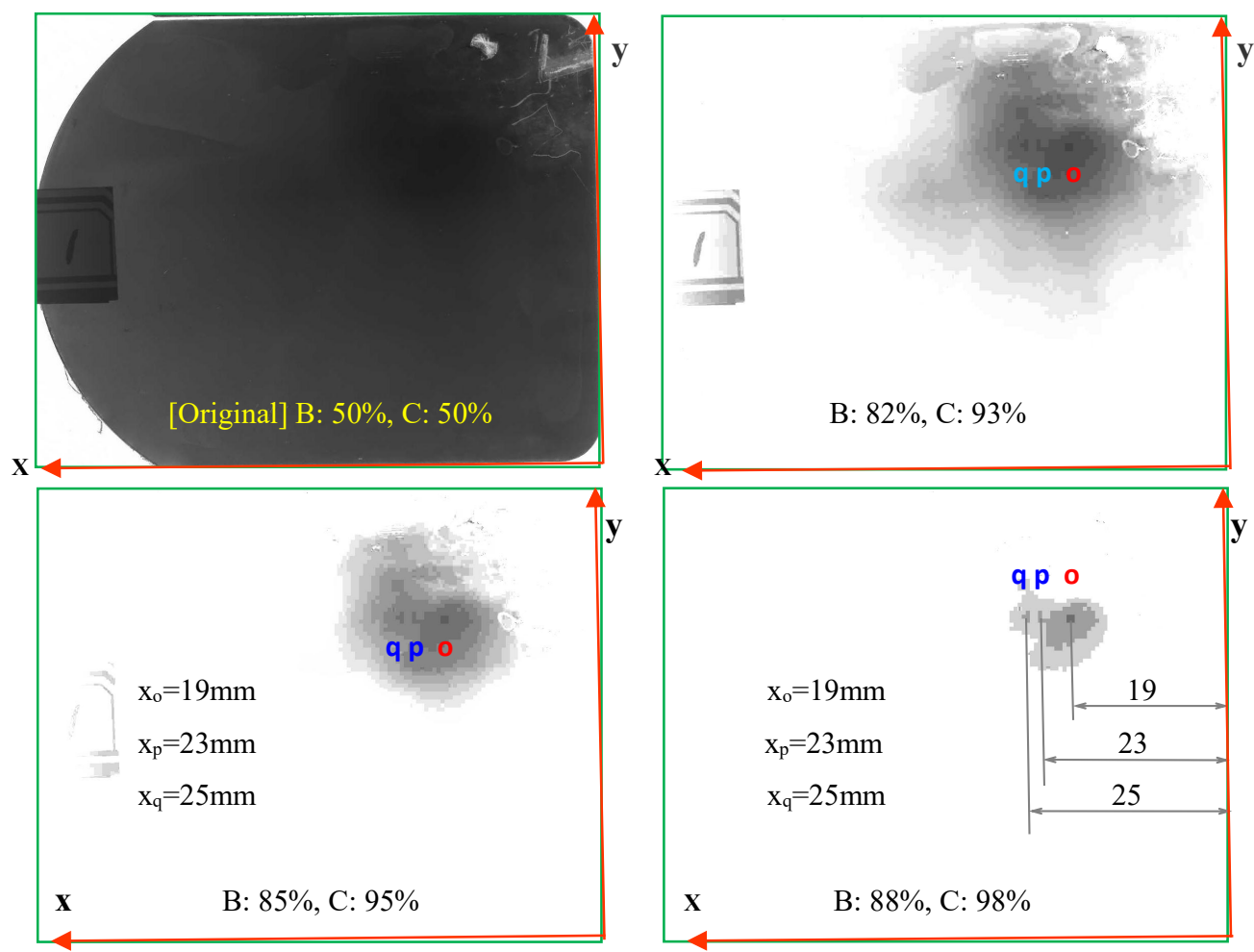

Figure 6. The \#1 film under the different brightness (B) and contrast (C)

The radiation from the straight section of north outer ring of Zone 1 of BEPCII emitting to the downstream copper blind end include the residual gas bremsstrahlung (GB), the X-ray in the synchrotron radiation and RF cavity, and other normal low energy radiation, and the predicted ultra-high energy electrons. The energy and intensity of the X-ray and synchrotron radiation are lower, and they have no the specific single emission angle. The \#1 film is widely photosensitized by the shower particles in the copper blind end caused by all radiations (See the original film under $50 \%, \mathrm{C}: 50 \%$ ).

Through properly increasing the brightness and contrast, those low-density photosensitive spots formed by shower particles scattered at large angles are filtered out, three prominent point-like photosensitive spots, o, p, q, are apparent on the \#1 film, in which the spot o is the most black, spots $p$ and $q$ are at the second level of high blackness. For the causes of forming three black spots, the analysis is as follows:

(1) As described in $\S 3.3 .1(\mathrm{i})$, the existence of the blackest point-shaped photosensitive spot shows that the electromagnetic shower in the metal absorber caused by the GB formed in the straight section is like a linear emission with a high density axis-line, although the electron beam is oscillating in the storage ring. 
During this experiment the electron beam changed two time of orbits, however, the two orbits are very close $(|\Delta \mathrm{XPOS}|<0.6 \mathrm{~mm})$ and the oscillation amplitude of electron beam is very small $(\sim 3 \mathrm{~mm}$ in the length range of $\sim 9 \mathrm{~m}$ ), so by the experience gained in $\S 3.3 .1$, the electromagnetic showers in the copper blind end caused by all GB will forms a high density axis-line. Further based the XPOS of beam and the fact that the affect of GB much strong than the ultra-high energy elctrongs in the early stage of develpement of electromagnetic shower, it can be inferred that the blackest spot o on \#1 film is the core of electromagnetic shower in the copper caused by all GB in $77 \mathrm{hrs}$.

(2) The theoretically predicted ultra-high energy electrons will be generated when the electron beam passes through the accelerating electric field of RF cavity. According to three XPOS of electron beam and the position of RF cavity, it can be inferred that the ultra-high energy electrons will towards the north direction slightly deviate from the axis-line of GB, and the deviation of the ultra-high energy electrons accumulated in $17.3 \mathrm{hrs}$ is slightly larger than that of the ultra-high energy electrons accumulated in $59.7 \mathrm{hrs}$. So it can be further inferred that electromagnetic showers in copper caused by two strands of ultra-high energy electrons have two cores, and form two photosensitive spots $p$ and $q$ on \#1 film, in which the spot $p$ is formed by the ultra-high energy electrons generated within $59.7 \mathrm{hrs}$, and the spot q is formed by the ultra-high energy electrons generated within $17.3 \mathrm{hrs}$ (See the "exaggerated schematic diagram" at the bottom of Fig.5).

(ii) The photosensitive spots on the films in the deep of lead layer appear abnormalities

According to the existing knowledge, since the electromagnetic shower caused by GB is the strongest, on all subsequent films, the photosensitive intensity of the spot corresponding to the position of o point on the \#1 film will always exceed the photosensitive intensity of other places, including at the positions of $p$ and $q$. However, the actually detected results are not the case, it is found through observation that after $\# 33$ film, i.e. after $\mathrm{Pb}$ depth $\sim 90 \mathrm{~mm}$, the photosensitive spot with $\mathrm{o}$ as the core is no longer stronger than the photosensitive spots with $p$ and $q$ as the core, but is that the latter exceed the former.

This phenomenon which is contrary to common knowledge is just consistent with the theoretical prediction of the existence of ultra-high energy electrons. Fig. 8 gives respectively the simulated curves of longitudinal distribution of electromagnetic showers in detector lead caused by the ultra-high energy electrons and GB, it can be seen that a series photosensitive features presented on films are in agreement with the simulation.

It is clear that there are three prominent black spots juxtaposed on every one of films after \#34. Their each other spacing is the same as that of the three spots on the \#1 film, and the tilt angle $\alpha$ of the three black spots to the z-axis of the detector is also the same, they are $\alpha=1.15 \pm 0.01^{\circ}$, which indicates that the three black spots on these films correspond to the o, $p$ and q points on the \#1 film, which record the distribution of three electromagnetic showers from the $\mathrm{Pb}$ depth of $\sim 92 \mathrm{~mm}$ to $\mathrm{Pb}$ depth of $\sim 120 \mathrm{~mm}$, that is, the size of black spot is $p+q>0$ or even alone $p>0$ on each film.

See Fig.7, the tilt angle $\alpha$ is calculated by the formula

$$
\alpha=\operatorname{arctg} \frac{\left|\mathrm{x}_{\# \mathrm{~N}}-\mathrm{x}_{\# 11}\right|}{(\mathrm{N}-1) \times 3.3}
$$

Where, $x_{\# 1}(\mathrm{~mm})$ is the coordinates of one point on the \#1 film, $x_{\# N}(\mathrm{~mm})$ is the coordinates of point that the electromagnetic shower axis-line extending from that point on the \#1 film falls on the \#N film. The unit thickness of $[\mathrm{Pb}+$ film and its sealing material $]$ is $3.3 \mathrm{~mm}$ (See Fig.2). 


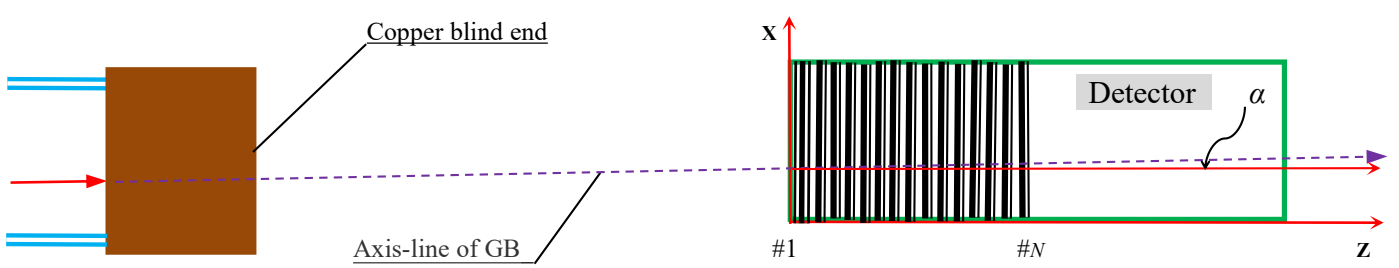

Figure 7. The skew angle $\alpha$ between the axis-line of electromagnetic shower and the coordinate $\mathrm{z}$-axis of detector

It is impordent that on all films after the \#33 the blackness and area of the photosensitive spots with the $p$ and $q$ as the core exceed those the photosensitive spots with the $o$ as the core. Especially on films $\# 41$ and $\# 42$ the spots $p+q$ exceeds the spot o by about four times the area, which is completely opposite with the situation of $0>>p+q$ on film \#1. This opposite phenomenon of the photosensitive situation is consistent with the simulation result based upon theoretical formulas, it can be seen in Fig. 8 that the blue curve simulating the electromagnetic shower caused by ultra-high energy electrons and red curve simulating electromagnetic showers caused by GB intersects at the lead thickness $\mathrm{z}=87 \mathrm{~mm}$ (between films \#32 and \#33), hereafter the blue curve exceed the red curve. This agreement between theoretical predictions and measured results provides important evidence for the existence of ultra-high energy electrons.

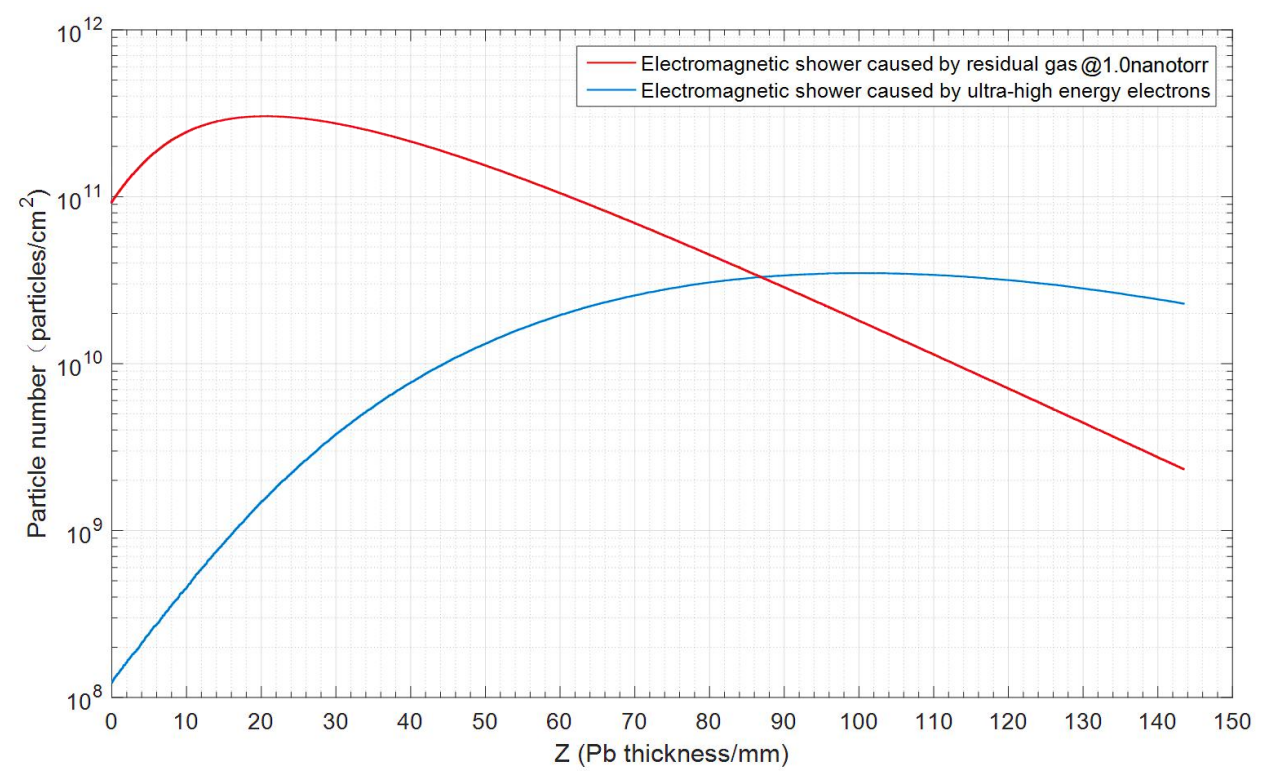

Figure 8. Simulation of the longitudinal distribution of the electromagnetic showers in the detector lead caused by the gas bremsstrahlung (GB) and ultra-high electrons generated during $59.7 \mathrm{hrs}$

In addition, on \#27 film there is a blackst photosensitive spot with an area $2 \mathrm{~mm} \times 2 \mathrm{~mm}$, its $\mathrm{x}$-coordinate of peak point is $25 \mathrm{~mm}$, the tilt angle to the detector coordinate $\mathrm{z}$-axis is $\alpha=1.14^{\circ}$, which shows that it corresponds to the $p$ spot on $\# 1$ film and so reflects the distribution at the lead thickness $72.8 \mathrm{~mm}$ of the electromagnetic shower caused by the ultra-high energy electrons generated during $59.7 \mathrm{hrs}$. There is a blackst photosensitive spot on \#26 film with an area $1 \mathrm{~mm} \times 1 \mathrm{~mm}$, its $x$-coordinate of peak point is $27 \mathrm{~mm}$, the tilt angle is also $\alpha=1.14^{\circ}$ to the z-axis, which shows that it corresponds to the q spot on \#1 film and so reflects the distribution at the lead thickness $70 \mathrm{~mm}$ of the electromagnetic shower caused by the ultra-high energy electrons generated during $17.3 \mathrm{hrs}$.

(iii) To display and analyze films under different brightness (B) and contrast (C) 


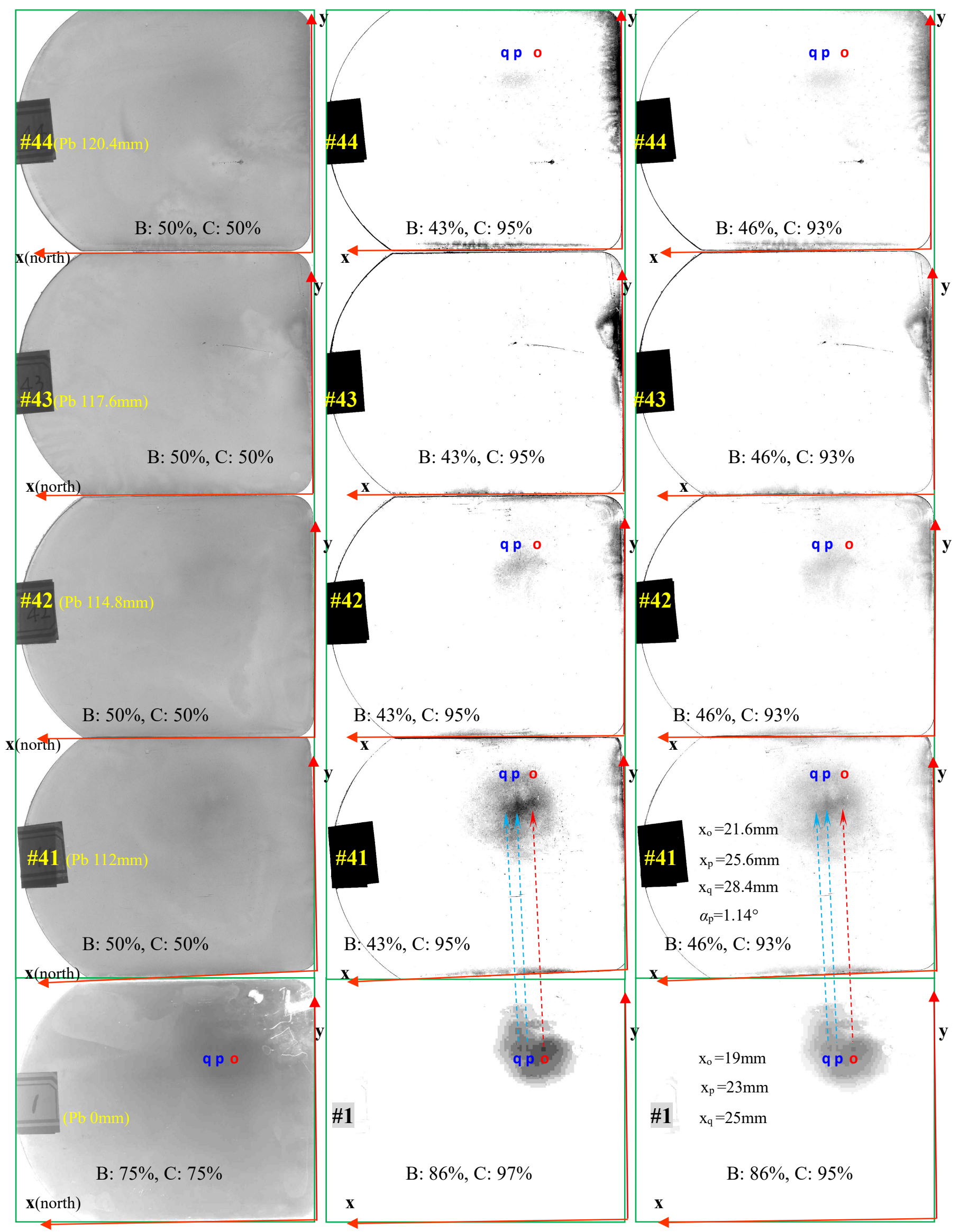

On films \#41 and \#42 the spot $p$ much exceeds the spot $\mathrm{o}$, which is completely reverse with the situation on film \#1. 
Such anomaly has shown on all films after \#34 film. Those are exactly what predicted by the theory.

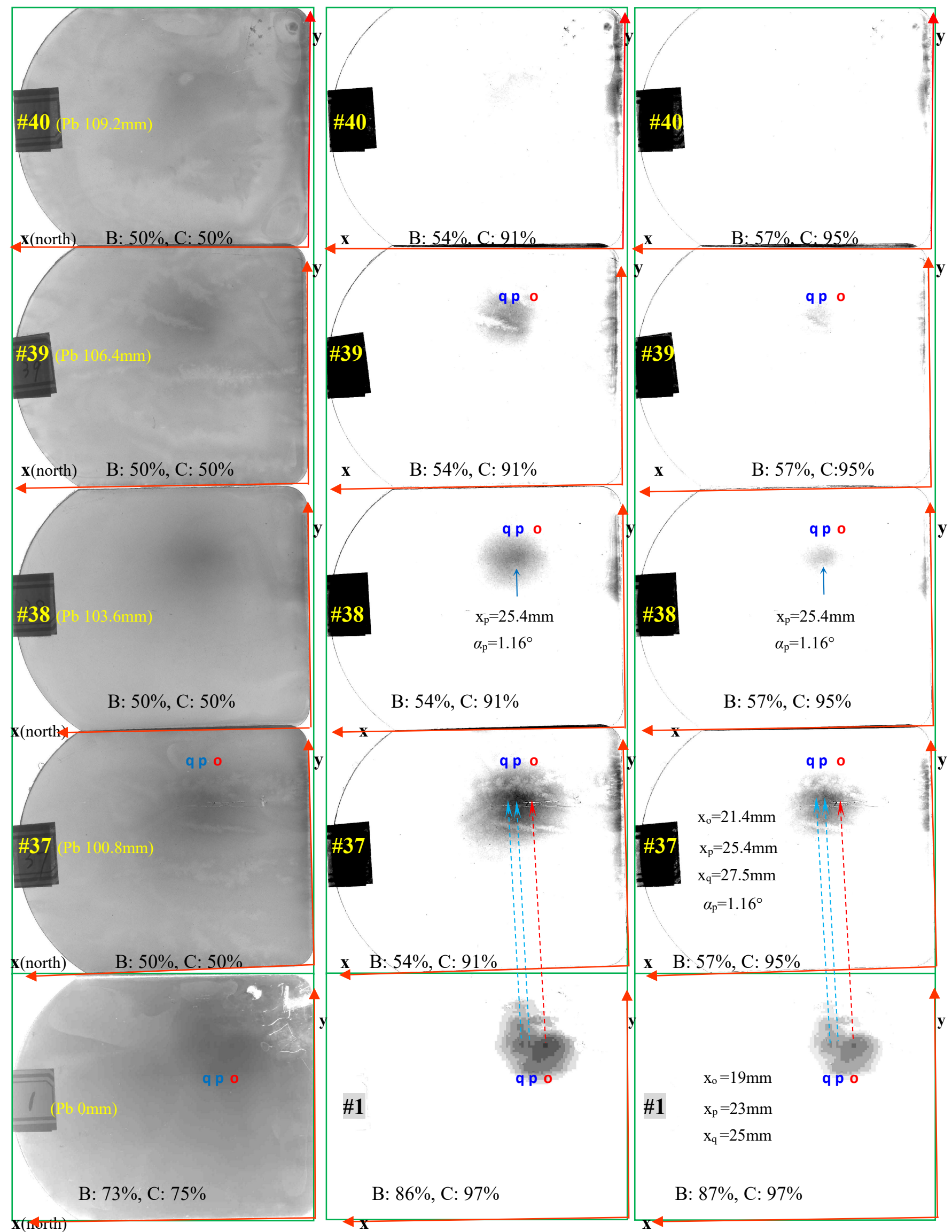




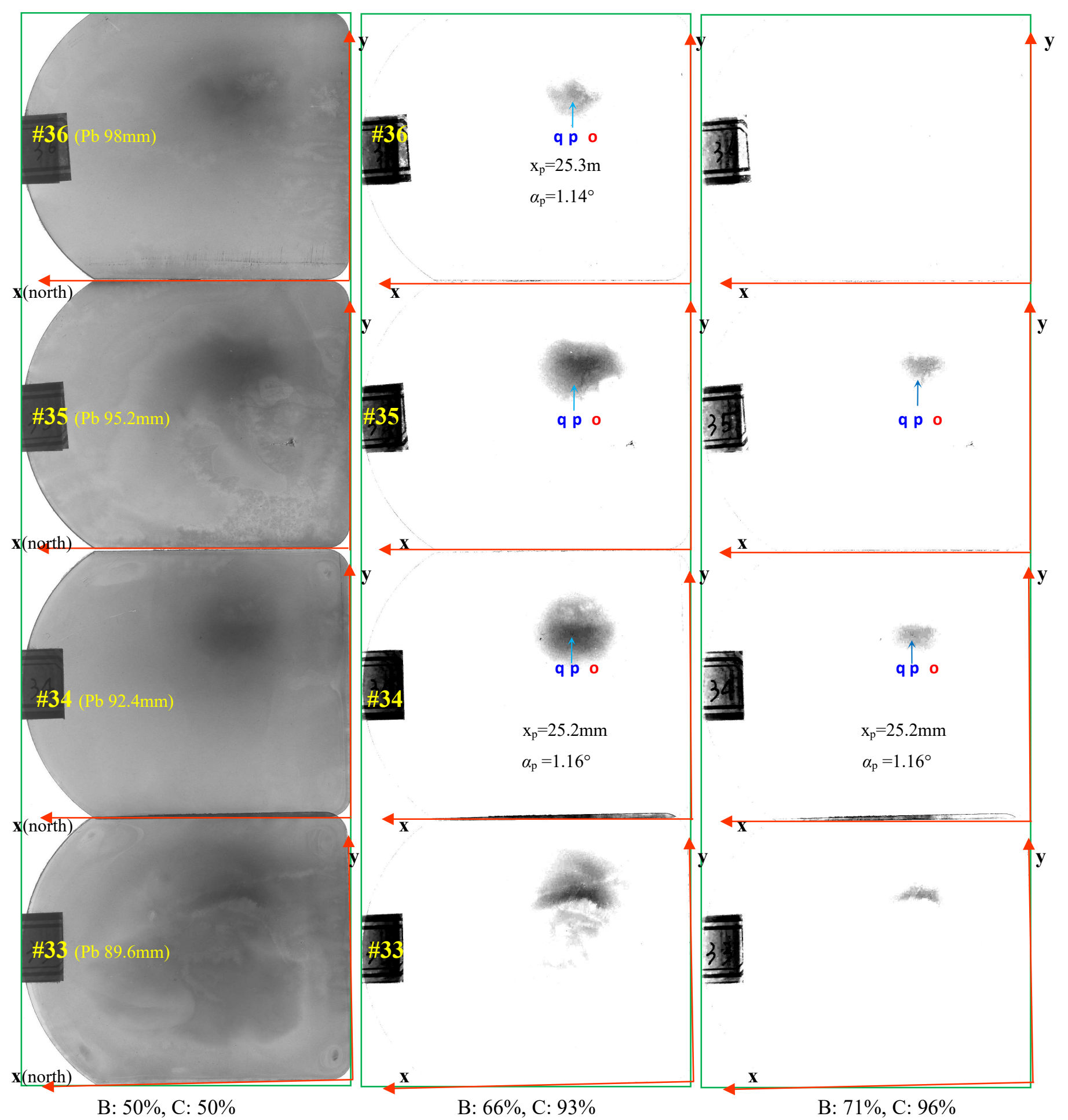

On every film after the $\# 34$ of $\mathrm{Pb}$ thickness $92.4 \mathrm{~mm}$, the size and blackness of the spot $\mathrm{p}$ all exceeds those of the spot $\mathrm{o}$, which fact in the error range is consistent with the simulations of the longitudinal distribution of electromagnetic showers in the lead caused by the ultra-high energy electrons and GB. It can be seen in Fig. 8 that the simulation curve (blue) for ultra-high energy electrons with the simulation curve (red) for GB intersects at the $\mathrm{Pb}$ thickness $87 \mathrm{~mm}$ that is between films \#32 and \#33, and hereafter the blue curve from low to high exceeds the red curve. 


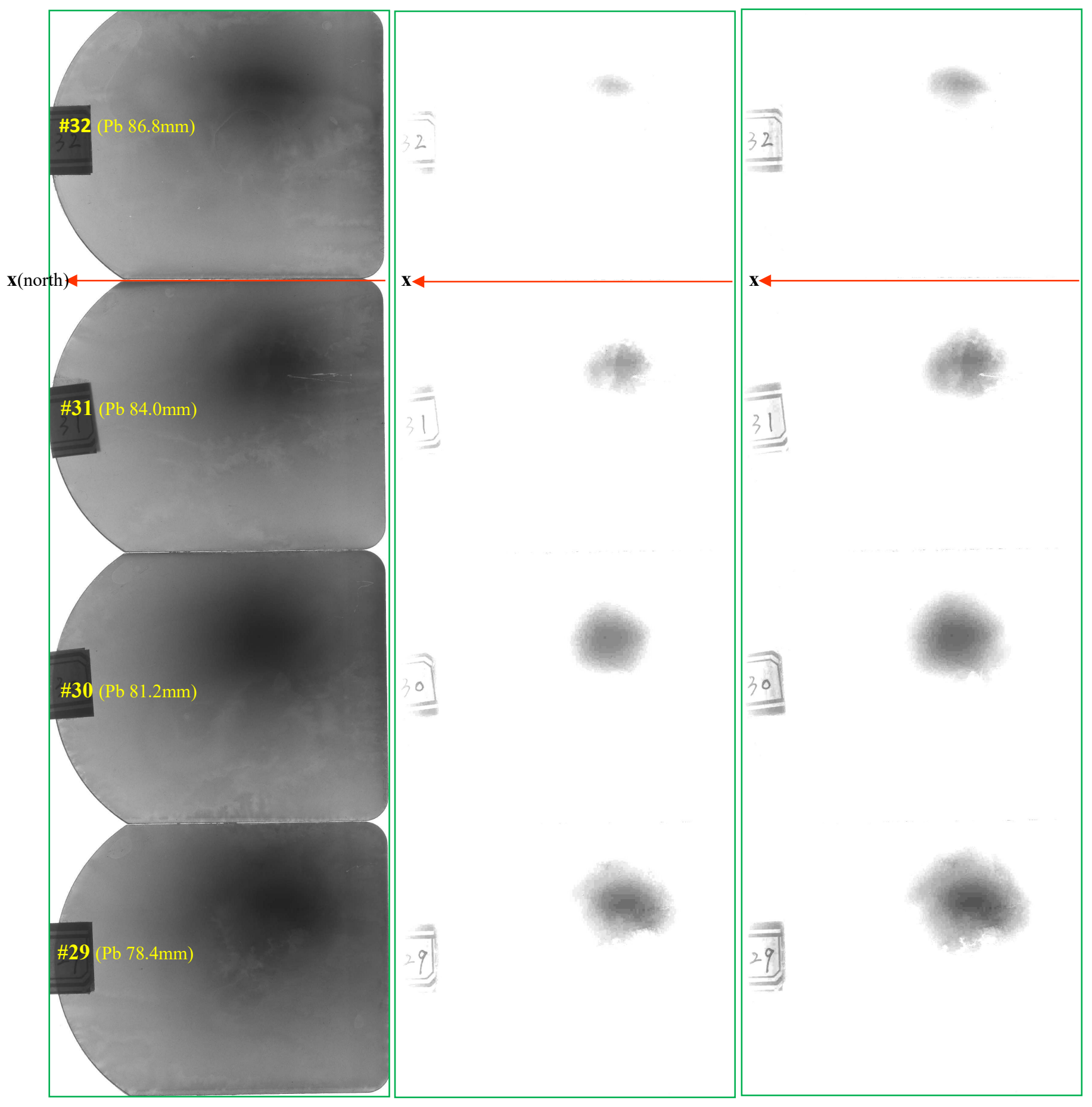

B: $50 \%, C: 50 \%$

B: $80 \%, C: 93 \%$

B: $79 \%, C: 92 \%$ 


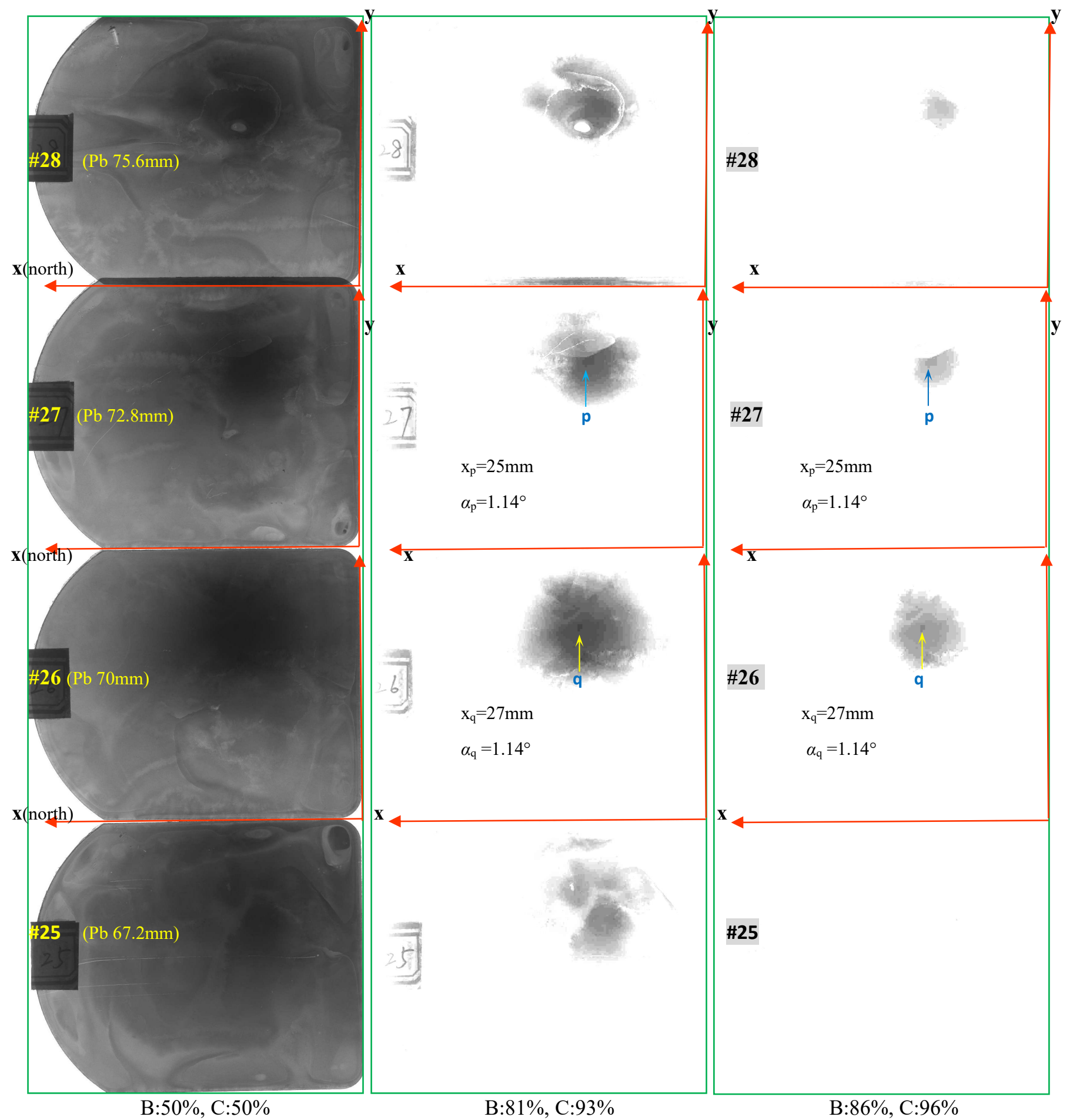

A high-blackness photosensitive spot appeared on \#27 film, according to the x-coordinate and tilt angle of its peak point, it can be judged that this is a continuation of $p$ spot on \#1 film. Also, a high-blackness photosensitive spot appeared on $\# 26$ film, according to the $x$-coordinate and tilt angle of its peak point, it can be judged that this is a continuation of q spot on \#1 film.

(\#25 film has photosensitive or processing problems, it loses the value of analysis) 
The photosensitive area on every film before \#25, that is before the lead thickness $67.2 \mathrm{~mm}$, is very large, which is a superposition result of a large number of secondary particles scattered in large angles. In this early stage of the development of electromagnetic shower, the shower core is covered by chaotic photosensitive spots in high-density, and the affect of ultra-high energy electrons is much lower than the affect of GB. This phenomenon is also consistent with the theoretical prediction.

Films \#1 to \#24 are listed below by B: $84 \%$ and C: $97 \%$ :

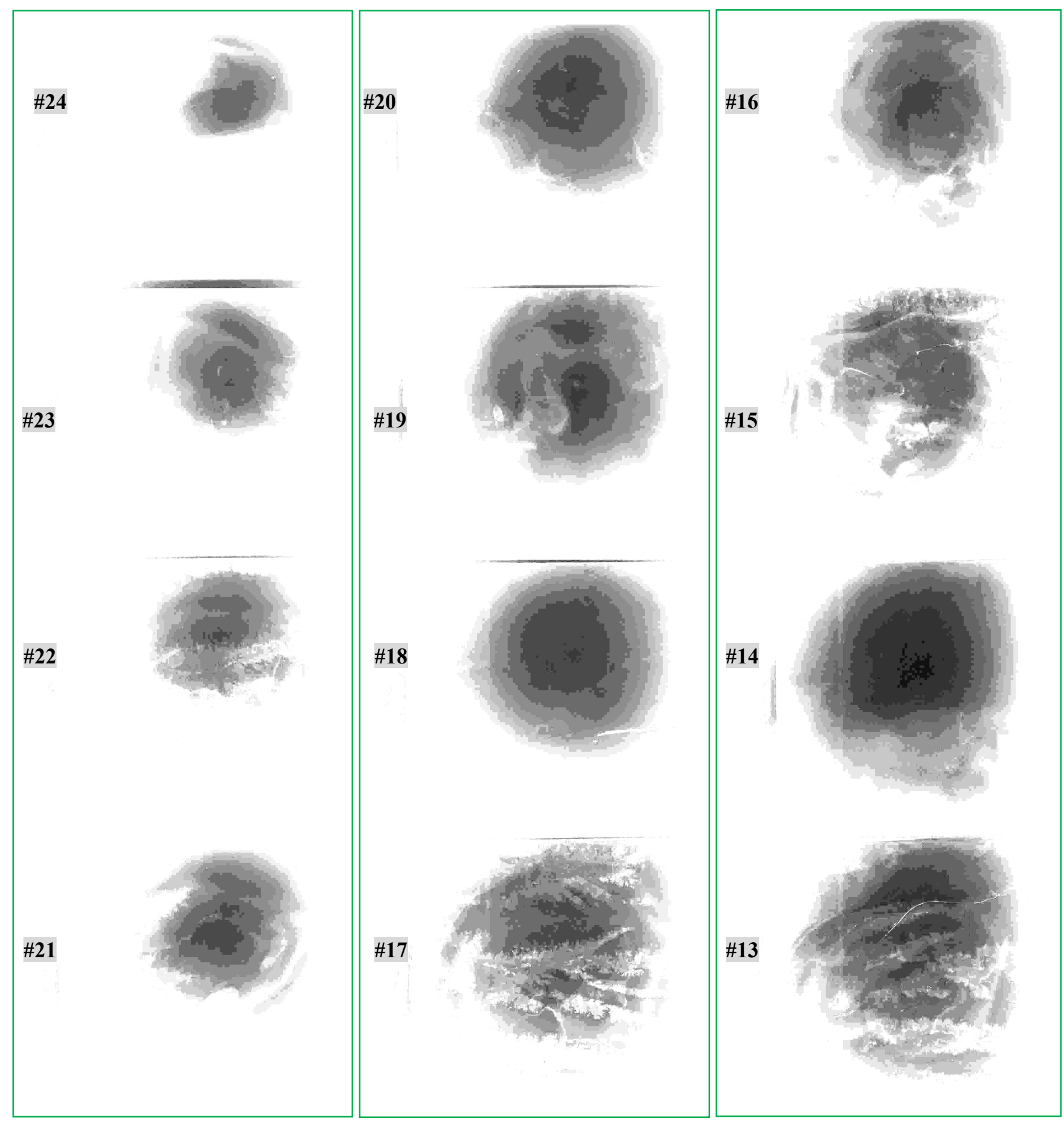




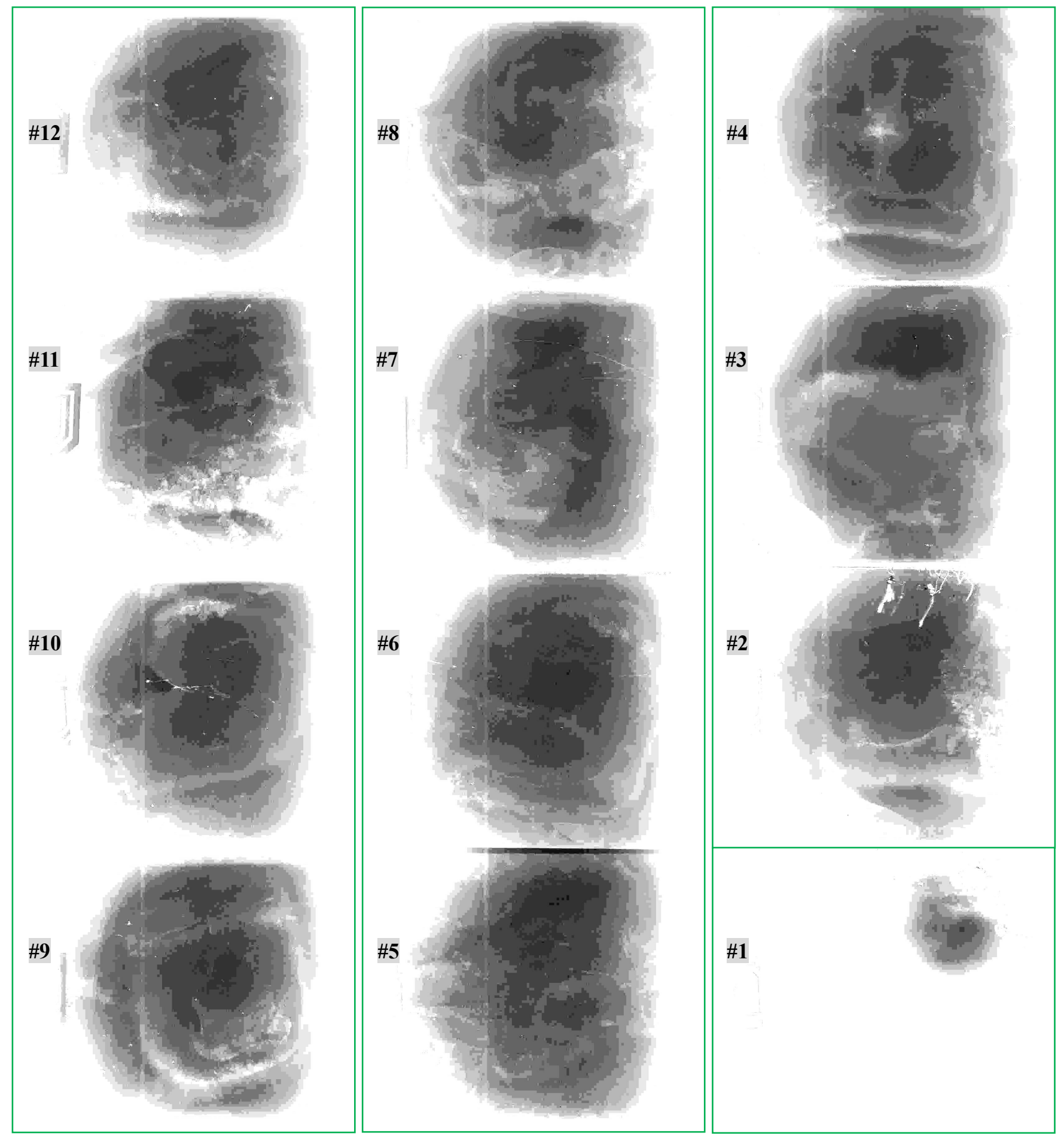

\section{Conclusion}

In a new version of special relativity that absorbed the uncertainty principle, the EinsteinLorentz mass formula is extended to a more general equation, which predicts that there is a bizarre "high speed and low mass (HSLM)" effect in the motion of particles. When an electron beam passes through an accelerating electric field, the HSLM effect will with a certain probability cause the generation of abnormal ultra-high energy electrons whose energy are much high than the beam. In order to verify the new theory, the author carried out experimental detection at BEPCII to this previously unknown phenomenon. 
Because the ultra-high energy electrons emit from the RF cavity of the electron storage ring toward the downstream copper blind end and cause electromagnetic showers in the copper, if there are indeed the ultra-high energy electrons, the electromagnetic calorimeter can detect abnormal electromagnetic shower caused by them that are different from various known effects. The experiment can be performed in two ways, of which one is to detect a single abnormal shower event caused by a single ultra-high energy electron, another is to detect a cumulative result of abnormal shower caused by a large number of ultra-high energy electrons during a long time.

The author's two experiments are the detection for cumulative result of a large number of events, by using a sampling electromagnetic calorimeter consisting of 48 sheets lead plate (2.8mm thick) and 49 sheets X-ray film. After a long for several days irradiation, the hierarchical distribution of cumulative shower in the lead pile of detector was recorded on a series of films. Films developed were input to the computer by the scanner, then to observe the photosensitive spots on films under the brightness and contrast appropriate adjusted by WPS software.

Fortunately, in the two sets of films in the two experiments, there appeared a series of independent photosensitive spots formed by a "pure" background. Therefore, through comparison with them, a series of abnormal photosensitive spots consistent with theoretical expectations were finally found, which provide evidence for the existence of ultra-high energy electrons.

In order to conduct more thorough verification, the author calls on the high energy physicists to do more experiments especially to use the electromagnetic calorimeter with an online real-time display function, such as the "Shashlic electromagnetic calorimeter", to detect the single event of electromagnetic shower caused by the single ultra-high energy electron.

In any case it is all interesting to do this experimental research, which is not only in order to look for a previously unknown strange phenomena, but also because the new theory that predicted this phenomenon has important science significance and application prospects. The new theory is an improvement and development to the special relativity under taking into account the universal constraints of the uncertainty principle, while the extended Einstein-Lorentz mass formula, as the most important result of the new special relativity, reveals that the concepts of mass and energy have richer connotations, which will not only can use to solve some unanswered problems but also help re-recognize the nature of certain phenomena and further discover new knowledge.

For examples:

i) Using the extended Einstein-Lorentz mass formula, a relationship formula between the Hubble constant and some basic constants can be derived, and so to calculate directly the theoretical value of the Hubble constant, which is in good agreement with a large number of observations ${ }^{[6][7][8]}$ :

$$
H_{0}=\frac{c G m_{\mathrm{e}} m_{\mathrm{n}}^{2}}{2 \hbar^{2}}=70.937 \mathrm{~km} \cdot \mathrm{s}^{-1} \cdot \mathrm{Mpc}^{-1}
$$

ii) It is pointed out that the existence of Lorentz invariance violation (LIV) is inevitable and the LIV coefficient $\xi$ can also be strictly calculated. For protons above $4 \times 10^{19} \mathrm{eV}$, the calculated $|\xi|<4.5 \times 10^{-30}<<10^{-23}$, which indicates that although there is LIV effect, it does not affect the GZK cut-off of the ultra-high energy cosmic ray, which is consistent with the observations of HiRes ${ }^{[9]}$ and Auger ${ }^{[10]}$.

iii) It is proved by the new formula that the Planck energy is a Lorentz invariant but is not the common upper limit of the highest energy that various particles can reach, which provides a basis for some BSM theories ${ }^{[1]}$ and also points out their shortcomings. 
iv) Since the HSLM effect allows the charged particles to more fully absorb energy in an accelerating electric field as to cause the generation of abnormal ultra-high energy particles at a lower accelerating voltage, so the problem of the acceleration mechanism of the ultra-high energy cosmic ray can be solved.

v) Because particles can appear in any state of $m_{R} \geq m \geq 0$ according to $1 \geq \zeta \geq 0$, so-call "zero-mass quasi-particle" in the condensed matter physics reflects the behavior of electron at $\zeta=0$ state.

vi) Electrons with energies exceeding $\mathrm{TeV}$ can only be detected in cosmic rays before, and they only appear as rare "signals". Once "mass production" of such ultra-high energy positrons and electrons in the laboratory will be used not only for ultra-high energy physics experiments, but also as an ultra-high energy radiation source for research in materials, medicine, life sciences, etc. contribution.

vii) Finally, it is pointed out that Eq.(1) is a part of the extended Einstein-Lorentz mass formula, which is applicable for interval $0 \leq u \leq u_{d}=\left(1-4.64 \times 10^{-39}\right) c$. The complete equation is as follows:

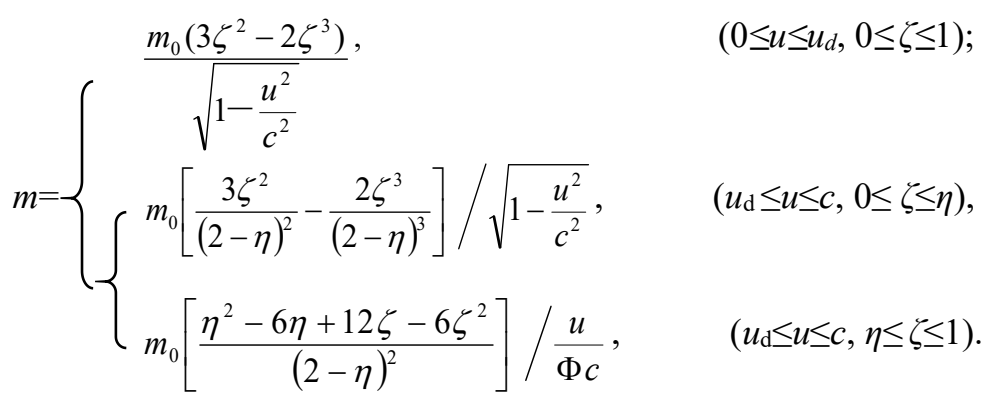

Where,

$$
\eta=2 \sqrt{1-\frac{u^{2}}{c^{2}}} /\left(\sqrt{1-\frac{u^{2}}{c^{2}}}+\frac{u}{\Phi c}\right)
$$

The extended Einstein-Lorentz mass formula indicates that the "instant mass" $m$ is a binary function of the speed $u$ and dimensionless variable $\zeta$, after weighting according to the probability of $\zeta$ state to get the "average mass" formula that is only depend on the speed $u$ as follows:

$$
\bar{m}=\frac{m_{0}}{\sqrt{1-\frac{u^{2}}{c^{2}}}+\frac{u}{\Phi c}}
$$

It can see that the extended Einstein-Lorentz mass formula gives another significant result, that is, when the particle's speed $u$ equals light-speed $c$, its mass $m$ is not infinite, but a limited maximum:

$$
\begin{aligned}
& m_{\max }=1.5 \Phi m_{0}=1.555 \times 10^{19} m_{0} \\
& \bar{m}_{\max }=\Phi m_{0}=1.037 \times 10^{19} m_{0}
\end{aligned}
$$

And more generally, when $u=c$, according to Eq. (18) $\eta=0$, so Eq. (1") becomes

$$
m=1.5 \Phi m_{0}\left(2 \zeta-\zeta^{2}\right)
$$

The formula (22) indicates that the mass $m$ (and energy $E=m c^{2}$ ) of a particle moving at the speed of light oscillates between 0 and $m_{\max }=1.5 \Phi m_{0}$ (and energy $E_{\max }=1.5 \Phi m_{0} c^{2}$ ) as the dimensionless random variable $\zeta$ changes in the range of $0 \leq \zeta \leq 1$.

\section{References}

[1] Qian Dapeng, A new version of special relativity absorbed the uncertainty principle: its content as well as application and experimental test, Frontier Science (Chinese version), Vol 5, No 1, 49-67 (2011), Journal of Modern Physics, 5, 1146-1166 (2014)

[2] Yang Chun, Li Yu-xion, Calculation of dose rate due to gas bremsstrahlung in the linear section 
of the electron storage rings, High Energy Physics and Nuclear Physics, Vol.28 No.5 (2004)

[3] Hua zheng-dong, Xu Xun-jiang, et al., Calculation of Gas Bremsstrahlung Power from Straight Section of Storage Ring at SSRF, Chinese Physics C (HEP \& NP), Vol. 32, (2008)

[4] Xie Yigang et al., Particle Detector and Data Acquisition, p27 and p315, Science Press (2004)

[5] Qian Dapeng, the signs of $38 \mathrm{GeV}$ electron emission detected on the $800 \mathrm{MeV}$ electron storage ring, Journal of Liaoning University (Natural Science Edition) 37, 236-240 (2010)

[6] R. L. Beaton1, W. L. Freedman, et al., The Carnegie-Chicago Hubble Program. I. An Independent Approach to the Extragalactic Distance Scale Using only Population II Distance Indicators (2016) [arXiv:1604.01788v2, accepted to ApJ (October 2016)]

[7] V. Bonvin, F. Courbin, S. H. Suyu, et al., H0LiCOW V. New COSMOGRAIL time delays of HE0435-1223: $H_{0}$ to $3.8 \%$ precision from strong lensing in a flat $\Lambda$ CDM model, (2017) [arXiv:1607.01790v2, accepted for publication in MNRAS]

[8] The LIGO Scientific Collaboration and The Virgo Collaboration, The 1M2H Collaboration, et al., A gravitational-wave standard siren measurement of the Hubble constant, Nature, 551, 85-88, (2017)

[9] HiRes Collaboration, First Observation of the Greisen-Zatsepin-Kuzmin Suppression, Phys. Rev. Lett. 100, 101101 (2008) [arXiv:astro-ph/0703099, accepted to PRL]

[10] Auger Collaboration, Recent results from the Pierre Auger Observatory, (2015) [arXiv:1503.09173]

[11] S.Coleman and S.L.Glashow, High-Energy Tests of Lorentz Invariance, Phys.Rev.D 59, 116008 (1999) [arXiv:hep-ph/9812418v3, Phys.Rev.D59:116008,1999] 


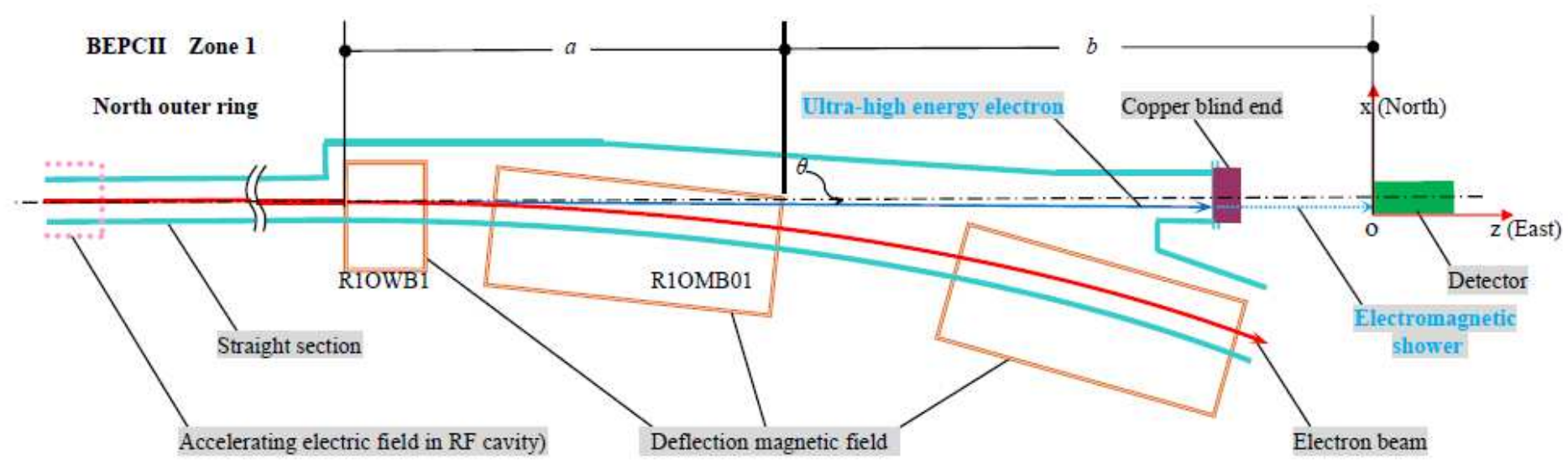

\section{Figure 1}

HSLM effect will cause the generation of abnormal ultra-high energy electrons with a certain small probability when the electron beam posses through the accelerating electric field

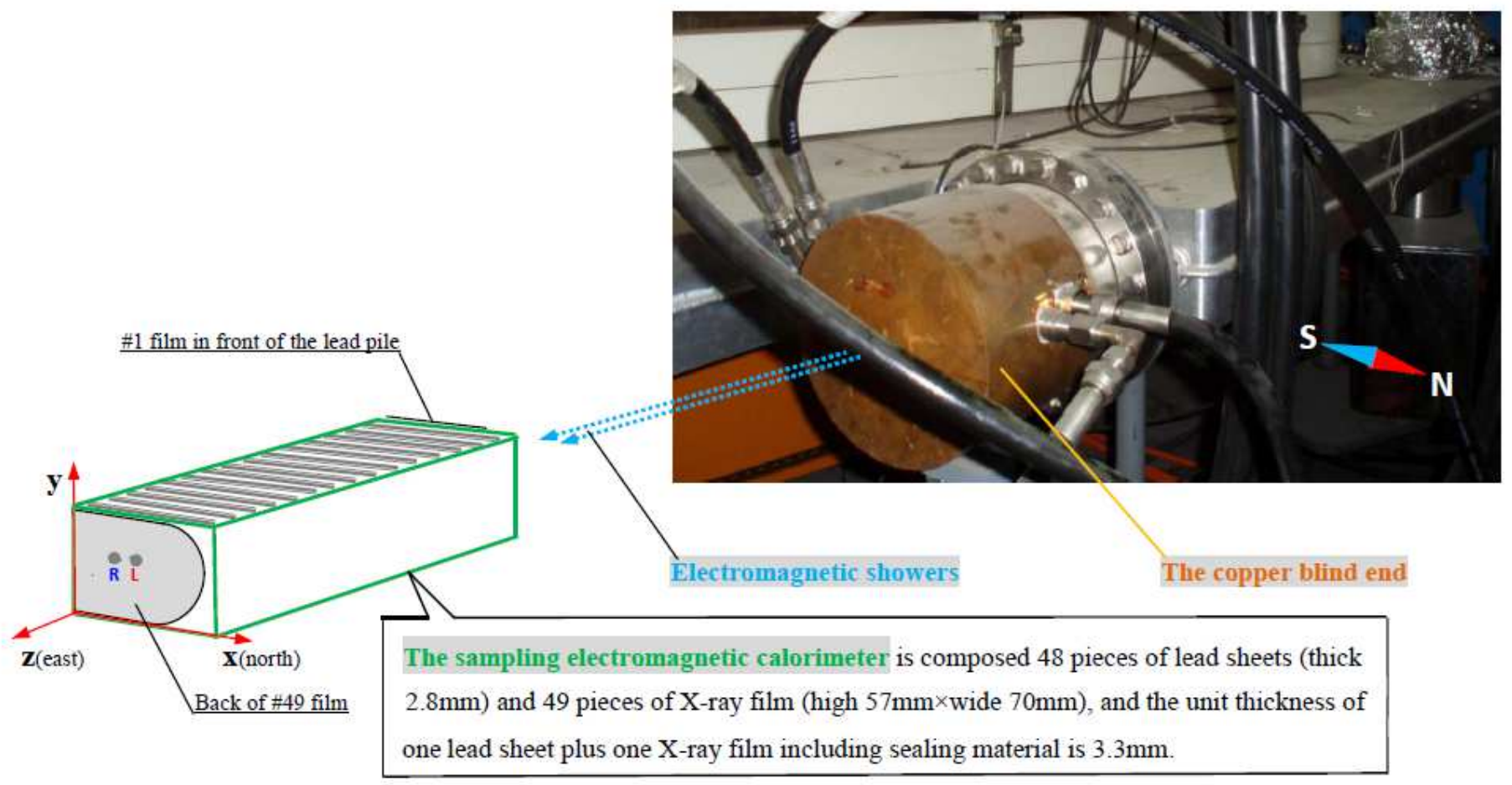

Figure 2

The copper blind end of downstream of the north outer straight section of zone 1 of BEPCII electron storage ring and the sampling electromagnetic calorimeter 


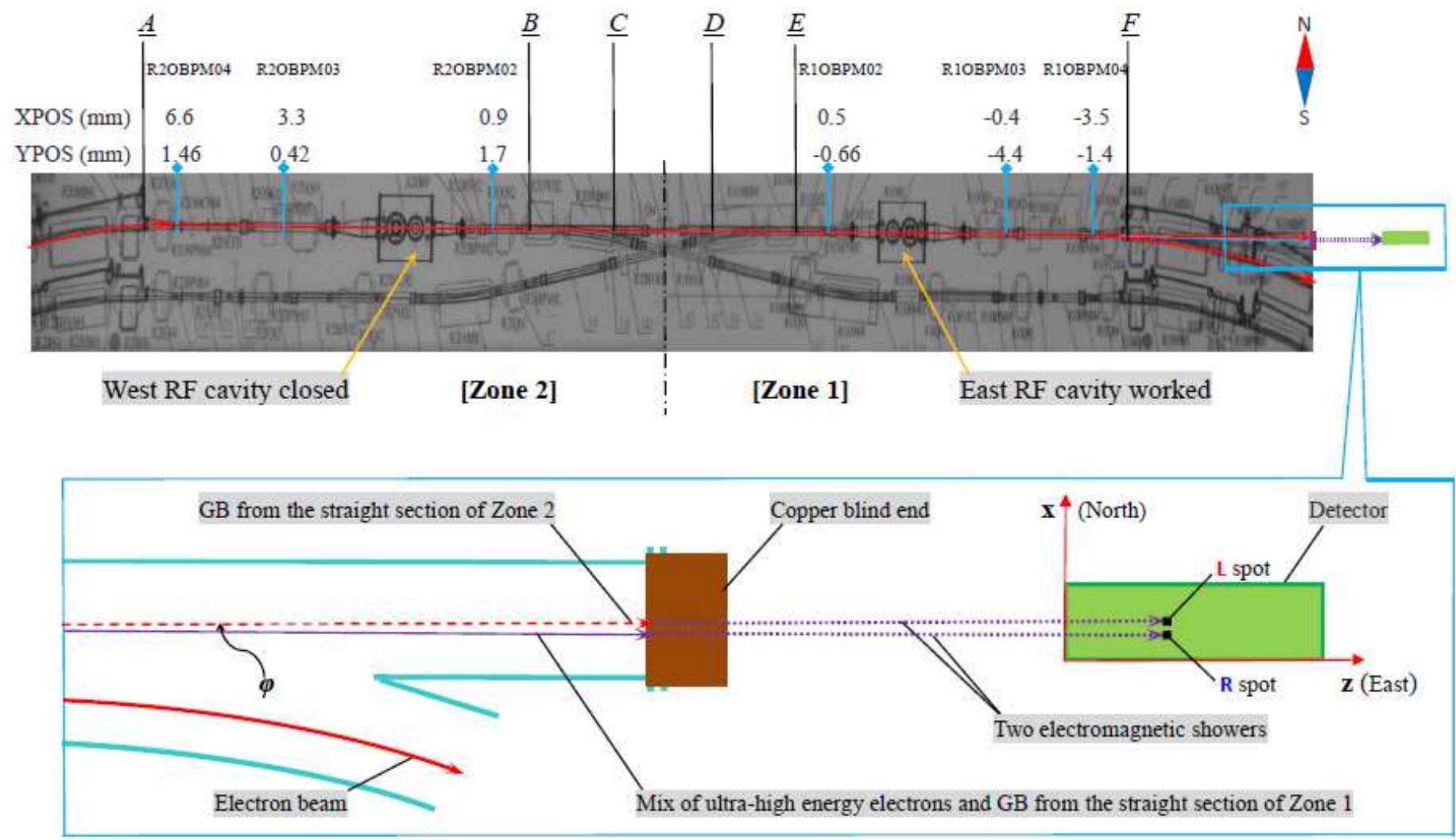

\section{Figure 3}

The gas bremsstrahlung (GB) and ultra-high energy electrons cause electromagnetic showers in the copper blind end of downstream of the east RF cavity of the electronic storage ring of BEPCII 


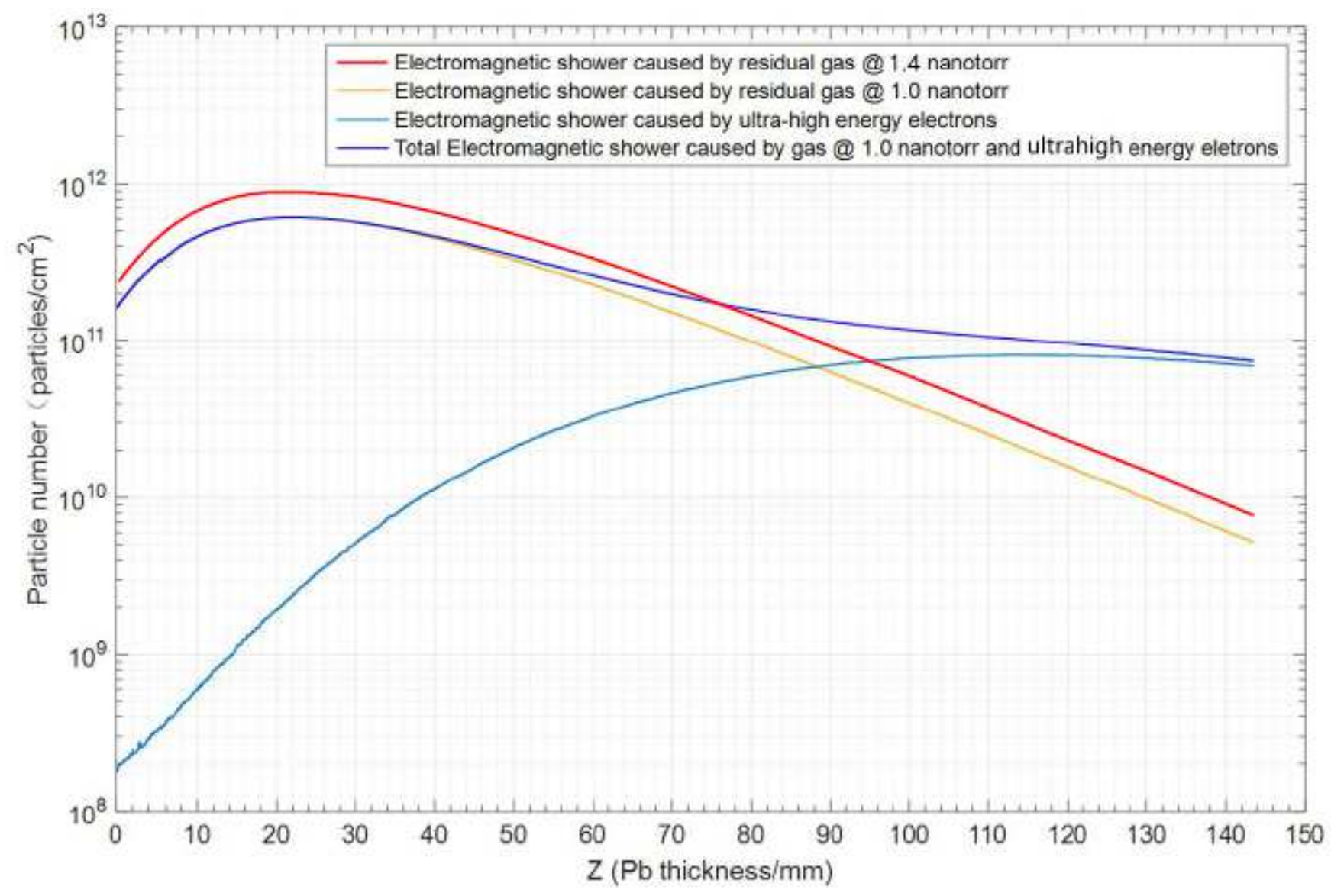

Figure 4

Simulation of the longitudinal distribution of the electromagnetic showers in the detector lead caused by three strands of radiation from two long straight section of the north outer ring of BEPCII 

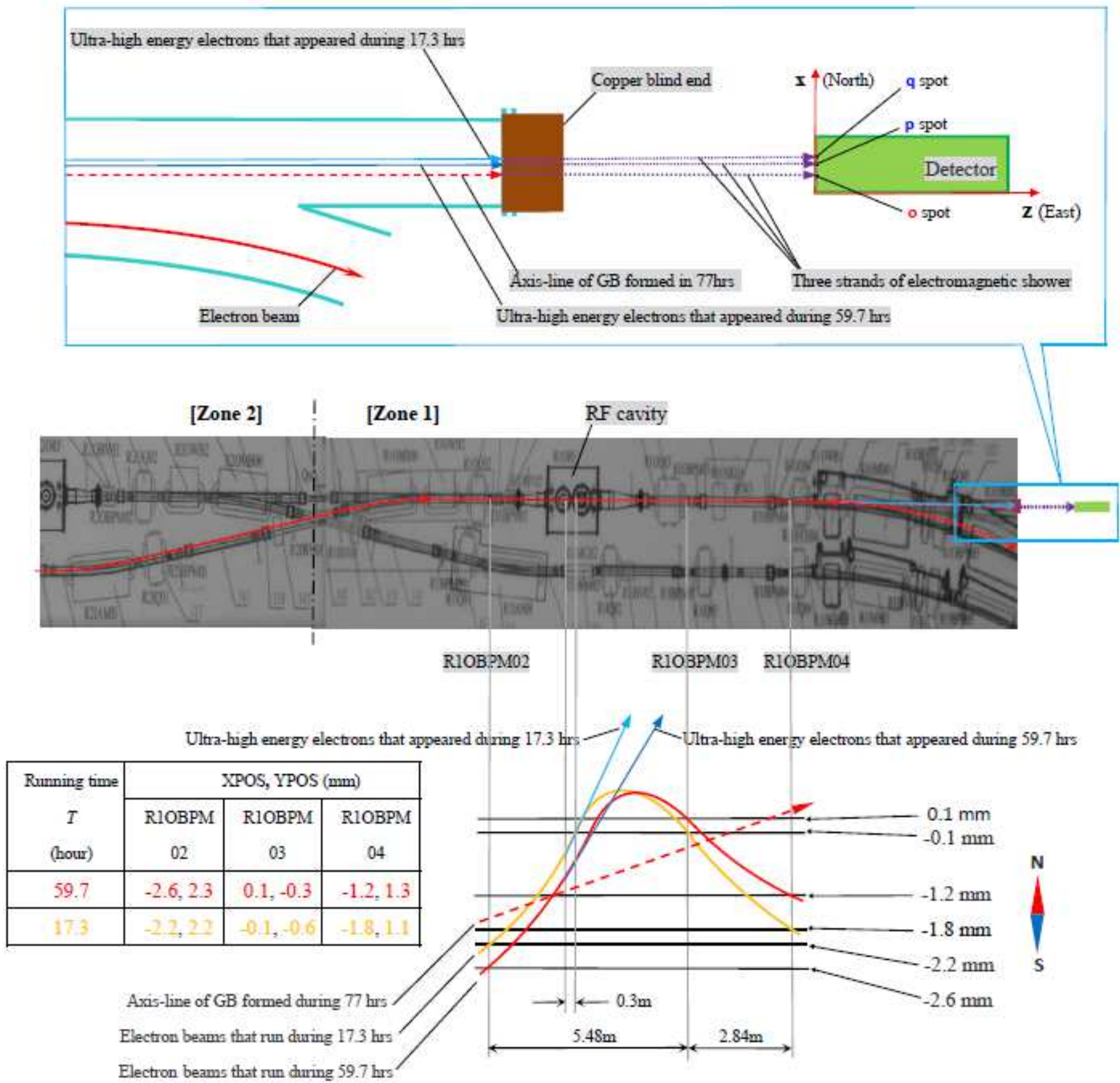

\section{Figure 5}

The operational parameters of electron beam and the enlarged schematic diagram of the emission direction of the ultra-high energy electrons and gas bremsstrahlung (GB) 

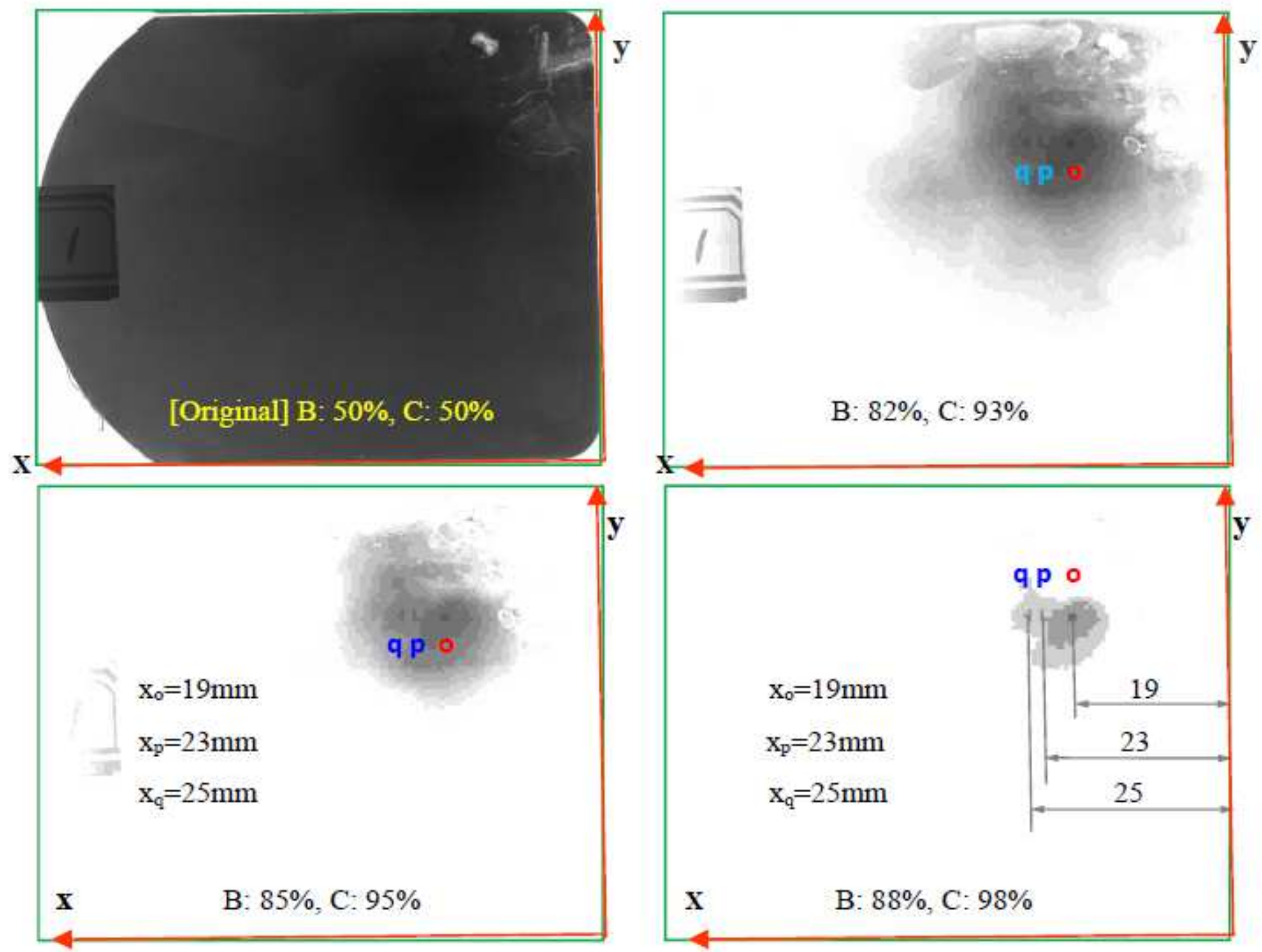

Figure 6

The \#1 film under the different brightness (B) and contrast (C)
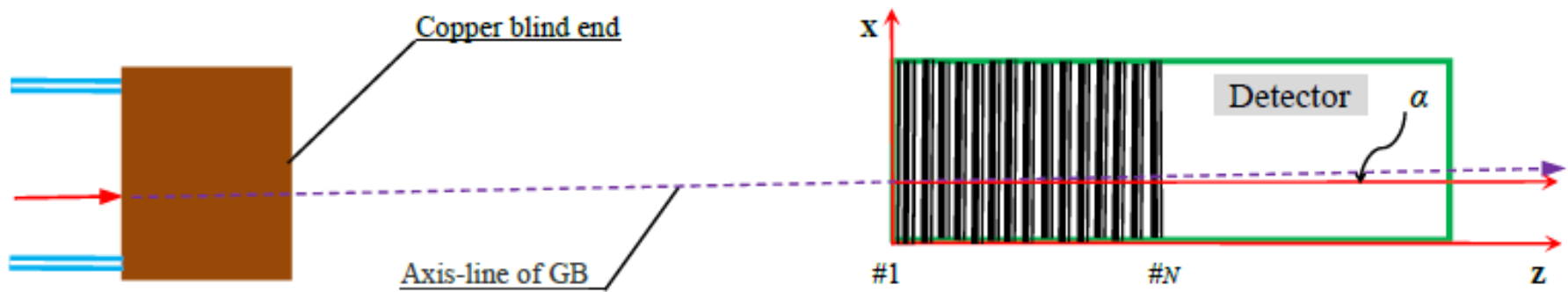

Figure 7

The skew angle a between the axis-line of electromagnetic shower and the coordinate z-axis of detector 


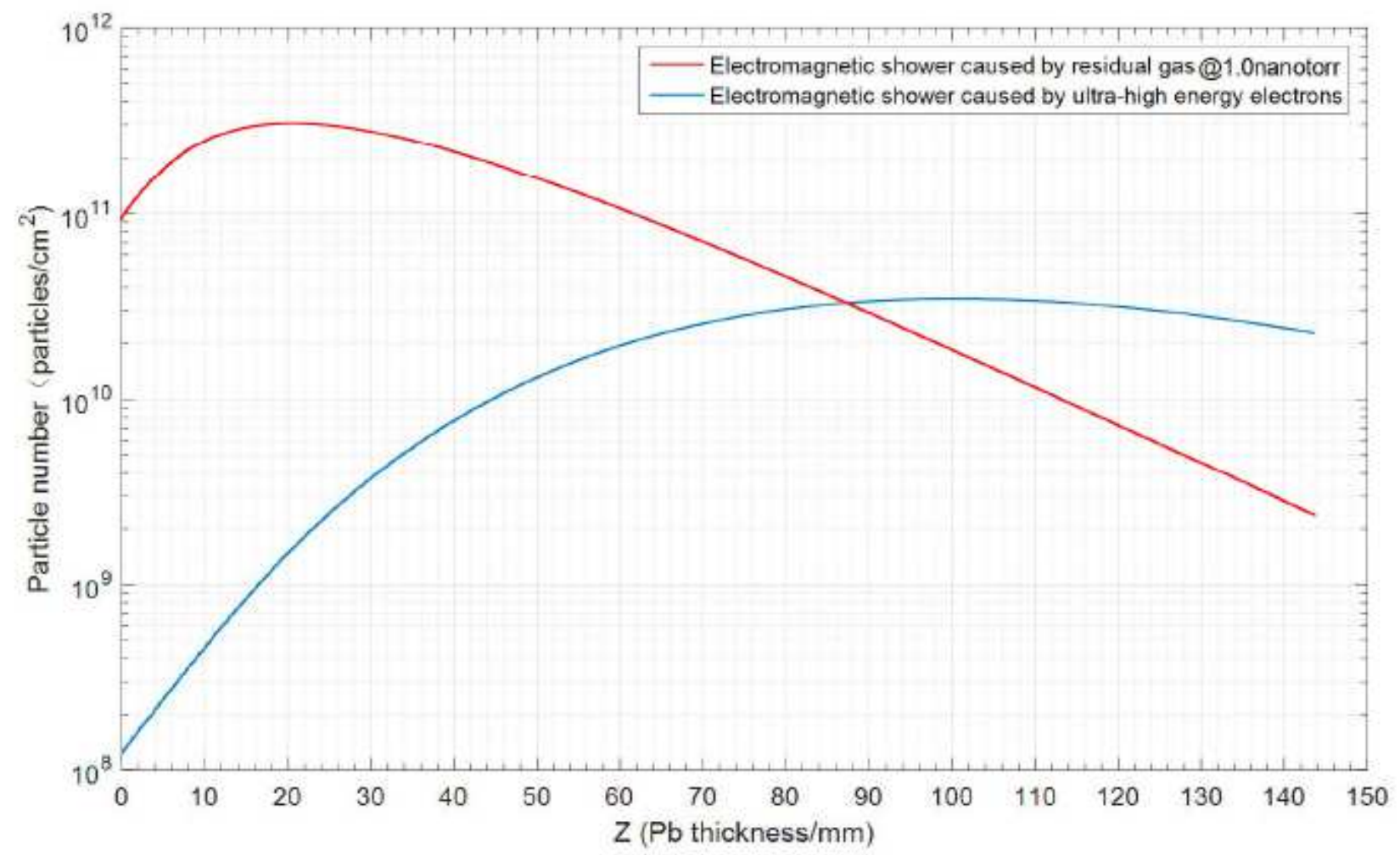

Figure 8

Simulation of the longitudinal distribution of the electromagnetic showers in the detector lead caused by the gas bremsstrahlung (GB) and ultra-high electrons generated during 59.7 hrs 